\title{
Overview of Repetition
}

\author{
MENYHÁRT László Gábor \\ (ORCID: 0000-0002-1574-4454) \\ ELTE Eötvös Loránd University, Budapest, Hungary \\ Faculty of Informatics, 3in Research Group, Martonvásár, Hungary
}

\begin{abstract}
There are differences between the everyday concept of repetition, the usage in informatics and the different implementations. This article collects the different levels, paradigms and language options and it presents a performed measurement and finally it evaluates the results didactically. So, it is useful for students preparing for a programming competition and their informatics teachers.
\end{abstract}

Keywords: repetition, recursion, iteration, programming languages, benchmark

\section{Introduction}

In the ordinary sense, repetition is a natural phenomenon: Think of regular alternations of days, weeks, seasons, etc. Nevertheless, it appears in IT in several ways. We can think differently, they occur differently in paradigms, they have different syntax and implementation in programming languages, so they run is different, so their run time can be very different. These observations and impressions have in-spired me to gather current opportunities and compare them.

This article reviews the conceptual definitions, measurement of running different implementations and didactic examination of the results. The presented method and the current results can be useful for students preparing for a programming competition and their informatics teachers.

\section{The concept of repetition}

Repetition is completion or execution of something several times. I collected some definition from different places, even though everyone knows or feels what it means.

Hungarian Synonym dictionary (Magyar Szinonima kéziszótár) contains the words: again (ismét) and repeat (ismétel):

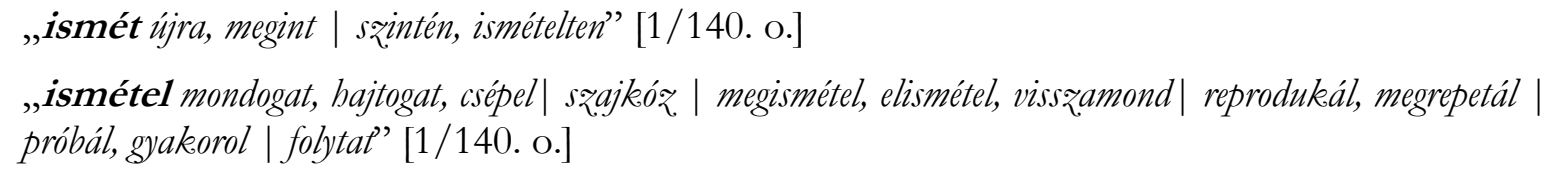

In New Hungarian Dictionary (Új Magyar Lexikon) the definition of repetition is this: „ismétlés :1. (nev) oktatási eljárás; ... Osz̧tály : ... 2. (nyelvt) ... A magyarban jelölheti a cselekmény hosszan tartó voltát (pl. ment-ment) ... 3. a stilisz̨tikában ..." [2/448. o.]

The Hungarian ethnographic lexicon (Magyar néprajzi lexikon) [3] provides only a linguistic definition to emphasize something to say as a poetic expression.

„ismétlés (fn.) ... Cselekvés, melynél fogva valamit ismét, azaz üjra, még egyszer teszünk. ...” can be found in [4].

Sulinet knowledgebase at Informatics subject contains definition of repetition as 'multiple process' („Többszöri végrehajtás”). [5]

Central-European Journal of New Technologies in Research, Education and Practice

Volume 2, Number 1, 2020. 
In WikiSzótár [6] one of the definitions of repetition is 'Redoing an action when we do the same thing we did before.' („2. Egy cselekvés újra végzése, amikor ugyanazt tesszük, amit korábban már megtettünk.").

In Cambridge dictionary repeat is defined as „to happen, or to do something, more than once” [7], while iteration is defined as ,the process of doing something again and again, ...” [8].

\section{Repetition on levels of informatics}

When we have a specific problem to solve, we just know that something will have to be done several times. At the next abstract level of our thinking, we decide whether an operation that we would like to perform several times will be a function and it calls itself, which is recursion [10], or will be repeated cyclically. This distinction is already present as a paradigm, since functional programming provides only the former.

Running of loops can be distinguished as conditional and specified number of times.

There are two groups at this specified number of times execution. First has a loop variable as a counter which changes at repetition, while the other has a given variable with the item from the series. With the first version to get the items from a series is possible with a counter which will be an index of the elements, the second version gives back immediately a given element from the series to process.

Conditional loops can be divided into two groups, first version checks the conditional at first, second checks it at the end. The difference is that the statements will be processed at least once when the checking is at last, while when the checking is at the beginning it possible that the statements are not processed. Both versions' condition is a logical expression which defines whether the statements must be processed (again) or not. Loops can be grouped by happening based on the condition. Namely whether the execution will happen or not at true expression. So, it can define the condition of staying inside or exit.

\begin{tabular}{|c|c|c|c|c|c|c|}
\hline \multicolumn{7}{|c|}{ Repetition } \\
\hline \multirow{4}{*}{ Recursion } & \multicolumn{6}{|c|}{ Iteration } \\
\hline & \multicolumn{4}{|c|}{ Conditional } & \multicolumn{2}{|c|}{ Running a certain number of items } \\
\hline & \multicolumn{2}{|c|}{ Check first } & \multicolumn{2}{|c|}{ Check last } & \multirow[b]{2}{*}{ Counter } & \multirow{2}{*}{$\begin{array}{c}\text { Iteration on } \\
\text { elements of serie }\end{array}$} \\
\hline & $\begin{array}{l}\text { staying } \\
\text { inside }\end{array}$ & $\begin{array}{c}\text { exit } \\
\text { cond. }\end{array}$ & $\begin{array}{l}\text { staying } \\
\text { inside }\end{array}$ & $\begin{array}{c}\text { exit } \\
\text { cond. }\end{array}$ & & \\
\hline
\end{tabular}

Table 1.: Overview table of repetition

Syntax can modify this clear picture, because most programming languages do not support any of the above options. For example, exit condition must be defined in Pascal for the conditional loop where the condition will be checked at last, but in C-like languages staying inside condition must be defined. Usually staying inside condition must be defined in those conditional loops where the condition will be checked at first, but shell script in Linux there is syntax for defining exit condition. Indeed, all four types of condition must be defined. In $\mathrm{C}$ the for keyword can be used as loop which checks the condition at first, and there are break and continue keywords to end earlier the for loop which runs theoretically a certain number of items. Of course, with multiple nested for loops you have to use tricks to, so you should avoid this option. There is programming language

Central-European Journal of New Technologies in Research, Education and Practice

Volume 2, Number 1, 2020. 
(like Logo), where loop-variable is not present generally, it can be used only if it required (in REPEAT the variable REPCOUNT).

Not only keywords and syntax can be different in high-level programming languages, but the compiler can change the actually running byte code and its implementation. So, their runtime can be different. I have already implemented the solution of the same tasks in different languages [11, $12,13,14,15,16]$ and the runtime was diverse, it occurred to me to make a more accurate measurement.

\section{Description of measurement}

I wrote code for five-six different loop implementation in six programming languages. Then I collected the measured run times in milliseconds.

Programming languages were $\mathrm{C}++, \mathrm{C \#}$, Java, JavaScript, Pascal and Python.

I implemented recursion, loops with checking conditional at first, checking it at last, iteration with loop variables as counter and as item if the language supported. It was skipped in case of not supported. Sometimes iteration with items can be implemented in more ways, then these were merged into a group.

\subsection{Presentation of the environment}

The measurement ran on a computer with Intel® Core $^{\mathrm{TM}} \mathrm{i} 7-8750 \mathrm{H} 2.20 \mathrm{GHz}$ processor and $32 \mathrm{~GB}$ memory, which has 64 bit Windows 10 Pro operating system.

The $\mathrm{C}++$ compiler was mingw32-g++.exe with version 5.1 .0 which is default in the Code::Blocks 17.12 .

I used .NER Framework's compiler for C\# found on Windows 10, it is „Microsoft (R) Visual C\# Compiler version 4.8.3752.0"'.

Java compiler was the currently most recent OpenJDK published on $6^{\text {th }}$ of October 2019.

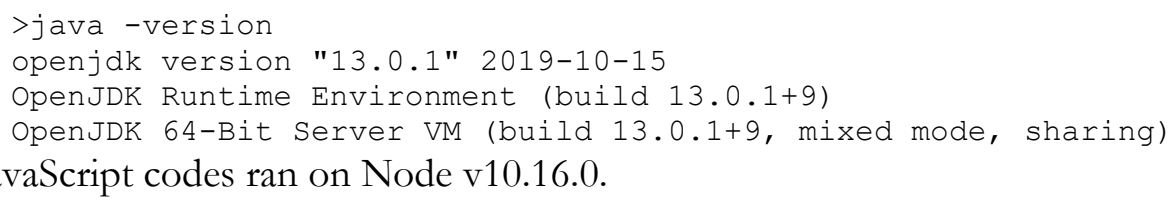

„Free Pascal Compiler version 3.0.4 [2017/10/06] for i386” was used for Pascal 32 bit.

Interpreter for Python codes was „Python 2.7 .15 (v2.7.15:ca079a3ea3, Apr 30 2018, 16:30:26) [MSC v.1500 64 bit (AMD64)]".

\subsection{Presentation of implemented algorithm}

Repetition was implemented in all six programming languages and possible syntaxes based on the next algorithm.

As found, number of steps is limited in recursion in most programming languages, at first the maximum number of steps was determined. Accurate values are in paragraph 4.3 in Table 2. This

Central-European Journal of New Technologies in Research, Education and Practice

Volume 2, Number 1, 2020. 
maximum value is not enough to measure all methods because it is too fast. So, codes must be run multiple times, (for) loop was chosen for this.

Elapsed time was calculated from the difference of start and end timestamp. Empty loop, namely loop without statements are not useable, so a simple assignment was present. But I was interested only in elapsed time of the loop, so I had to remove the time of the assignment. So, I implemented the same with two assignments as well. Abstract algorithm:

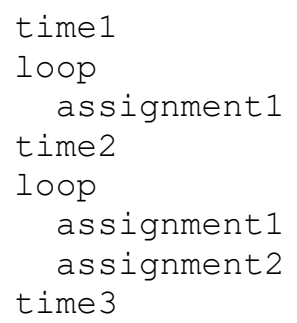

I calculated the total time devoted to an assigment (1) and I assumed that time of assignments are equals (2). I calculated (3) the specific time of the given loop:

$$
\begin{gathered}
T_{a 2}=\left(t_{3}-t_{2}\right)-\left(t_{2}-t_{1}\right) \\
T_{a 1}=T_{a 2} \\
T_{l}=t_{2}-t_{1}-T_{a 1}
\end{gathered}
$$

Implemented codes can be found in Appendix A.

\subsection{Results of measurement}

At first the maximum number of steps was determined based on the implementation of recursion which is good in all programming languages. The recursive function calls happen at the beginning of the function to avoid the optimization of tail-recursion.

\begin{tabular}{|c|c|}
\hline $\begin{array}{c}\text { Programming } \\
\text { language }\end{array}$ & $\begin{array}{c}\text { MaxCnt } \\
\text { (In this environment, with these program codes) }\end{array}$ \\
\hline C ++ & 56988 \\
\hline C\# & 15918 \\
\hline Java & 11420 \\
\hline JavaScript & 8940 \\
\hline Pascal & 65134 \\
\hline Python & 4194 \\
\hline
\end{tabular}

Table 2:: Maximum number of recursive function calls

Finally, 4000 repetition happened 10.000 times in an iteration with simple for syntax to get measurable and comparable results. Later it was detected that it was good choice because this is the fastest and that is why it does not cause confusion in comparison. So double times 40.000 .000 loops were measured.

Central-European Journal of New Technologies in Research, Education and Practice

Volume 2, Number 1, 2020. 


\subsection{Evaluation of measurement}

Appendix B. contains raw data of measurement from which average and relative deviation was determined with Excel. The time $\left(T_{1}\right)$ in milliseconds concern to the first 40 million loops. Deviation is given in relative to average in percentage, so it is easier to understand and compare. Next table contains the results:

\begin{tabular}{|c|l|l|l|l|l|}
\hline $\begin{array}{c}\text { Programing } \\
\text { language }\end{array}$ & \multicolumn{1}{|c|}{ While } & \multicolumn{1}{|c|}{ For } & $\begin{array}{c}\text { ForEach } \\
\text { [+lambda] }\end{array}$ & $\begin{array}{c}\text { ForEach } \\
\text { +named fnc }\end{array}$ & Recursion \\
\hline C++ & $7(127 \%)$ & $6(113,9 \%)$ & $1142(8,8 \%)$ & $1141(12,7 \%)$ & $135(17,7 \%)$ \\
\hline C\# & $20(46,8 \%)$ & $24(55,1 \%)$ & $117(43 \%)$ & $113(69,2 \%)$ & $90(10,2 \%)$ \\
\hline Java & $54(42,2 \%)$ & $50(21,6 \%)$ & $\begin{array}{l}234(22 \%)^{*} \\
170(18,7 \%)\end{array}$ & $2020(12,4 \%)$ & $109(15,9 \%)$ \\
\hline JavaScript & $36(37,5 \%)$ & $41(68,1 \%)$ & $254(18,6 \%)$ & $242(14,7 \%)$ & $267(9,2 \%)$ \\
\hline Pascal & $65(10,1 \%)$ & $89(36,5 \%)$ & $565(8,6)^{*}$ & notSupported & $138(11,3 \%)$ \\
\hline Python & $2054(28,8 \%)$ & $1156(55,3 \%)$ & $460(95,4 \%)^{*}$ & $3589(20,8 \%)$ & $9876(15,4 \%)$ \\
\hline
\end{tabular}

Table 3.: Results of measurement: average (deviation relative to the average in $\%$ )

\subsection{Opinions of the results}

You can see in the table that the specified number of steps with loop variable (for) and the conditional loop with checking at first (while) are the fastest repetition methods only there is deviation in Python. Recursion is the next which is slower double times in Pascal and Java, fourfive times slower in C\#, JavaScript and Python, and twenty times slower in $\mathrm{C}++$, as the averages of previous two. Previous average times are fraction of a second but in Python it is around 10 seconds. Similar time was measured for iteration over the items of series with C\# and JavaScript to the time of recursion calls, but it is nine times slower in $\mathrm{C}++$, and two times or eighteen times slower in Java depends on usage of anonymous or named functions at iteration. While it is "only" three times slower in Python compared to for loop, but it is faster than recursion. For loop in C++ is the fastest, and recursion is Python is the slowest.

\section{Didactic consideration}

If I had to choose a programming language for education based on the table above, I would choose $\mathrm{C \#}$, because the measured times are consistent. Pascal does not support every method, so it should be skipped. Python is too slow. So, my order would be C\#, JavaScript, C++ and Java based on the average runtime and its deviation.

Of course, all methods must be taught to the students and should be shown how they can choose between paradigms and syntaxes.

There are cases where the readability of the code is more important, but sometimes fast runtime is necessary. For instance, it was determined [9], that recursion calls usually less effective then iterations, but we often get a simpler, easier-to-read code. Such a comparative table can also be useful in programming competitions where time limits are used, and the competitor can choose 
the language. However, the statement is not only an assignment, and other language elements can affect the total runtime.

The knowledge and measurement results from this article can help you to choose the right implementation for a given task. The described technique can be helpful later if Benchmark [17] would be performed again because of processor development and appearance of newer compilers may change the times described here. Because newer compiler versions can analyse the codes and create more efficient binary codes with varying changes of codes. Like Python has version 3.8, and there are alternate implementations $[18,19]$ as well, which would be worth comparing.

The research has been supported by the European Union, co-financed by the European Social Fund (EFOP-3.6.2-16-2017-00013, Thematic Fundamental Research Collaborations Grounding Innovation in Informatics and Infocommunications).

\section{References}

1. Bék Gerzson, Csiffáry Tamás: Magyar szinonima kézişzótár, Könyvmíves Könyvkiadó, Budapest (2004)

2. Akadémiai kiadó szerkesztősége: Új magyar lexikon, 3 G-J, Nyolcadik, változatlan lenyomat, Akadémiai Kiadó, Budapest (1962)

3. Magyar néprajæi lexikon, Akadémiai Kiadó, Budapest (1977-1982)

4. Czuczor Gergely, Fogarasi János: A magyar nyelv sqótára, Emich Gusztáv at Hungarian academic printer Pest (1862)

https://czuczor.oszk.hu/kereses.php?kereses=ism\%C3\%A9tl\%C3\%A9s (last viewed:

2019.11.11.) and https://mek.oszk.hu/05800/05887/pdf/3kotet 1.pdf (page 74., last viewed: 2019.11.11.)

5. The concept of repetition in the Sulinet knowledgebase (2016)

https://tudasbazis.sulinet.hu/hu/informatika/informatika/informatika-2-

evfolyam/algoritmusok-a-hetkoznapokban-tevekenysegek-elemekre-bontasa-helyes-sorrendmegkeresese/ismetles-szerepe (last viewed: 2019.11.11.)

6. WikiS zótár Dictionary (2012) https://wikiszotar.hu/ertelmezoszotar/Ism\%C3\%A9tl\%CC3\%A9s (last viewed: 2019.11.11.)

7. Repeat in Cambridge Dictionary (2019) https://dictionary.cambridge.org/dictionary/english/repeat (last viewed: 2019.11.11.)

8. Iteration in Cambridge Dictionary (2019) https://dictionary.cambridge.org/dictionary/english/iteration (last viewed: 2019.11.11.)

9. Rónyai L., Ivanyos G., Szabó R.: Algoritmusok, Typotex (2004) page 37.

10.Thomas H. Cormen, Charles E. Leiserson, Ronald L. Rivest.: Introduction to Algorithms, MIT Press (1990) (Hungarian version: Algoritmusok, Múszaki Könyvkiadó 1997)

11.C++ reference (2019) https://en.cppreference.com/w/ (last viewed: 2019.11.11.)

12.C\# reference (2017) https://docs.microsoft.com/en-us/dotnet/csharp/language-reference/ (last viewed: 2019.11.11.)

Central-European Journal of New Technologies in Research, Education and Practice

Volume 2, Number 1, 2020. 
13.Java documentation (2019) https://docs.oracle.com/javase/tutorial/java/(last viewed: 2019.11.11.)

14.JavaScript reference (2019) https://developer.mozilla.org/hu/docs/Web/JavaScript/Reference (last viewed: 2019.11.11.)

15.Free Pascal reference guide (2017) https://www.freepascal.org/docs-html/ref/ref.html (last viewed: 2019.11.11.)

16.The Python Language Reference (2019) https://docs.python.org/2.7/reference/index.html (last viewed: 2019.11.11.)

17.Meaning of Becnhmark https://dictionary.cambridge.org/dictionary/english/benchmark (last viewed: 2019.12.26.)

18. Alternative Python Implementations https://www.python.org/download/alternatives/ (last viewed: 2019.11.11.)

19.Cython https://cython.org/ (last viewed: 2019.11.11.)

\section{Appendix}

\section{A. Source codes}

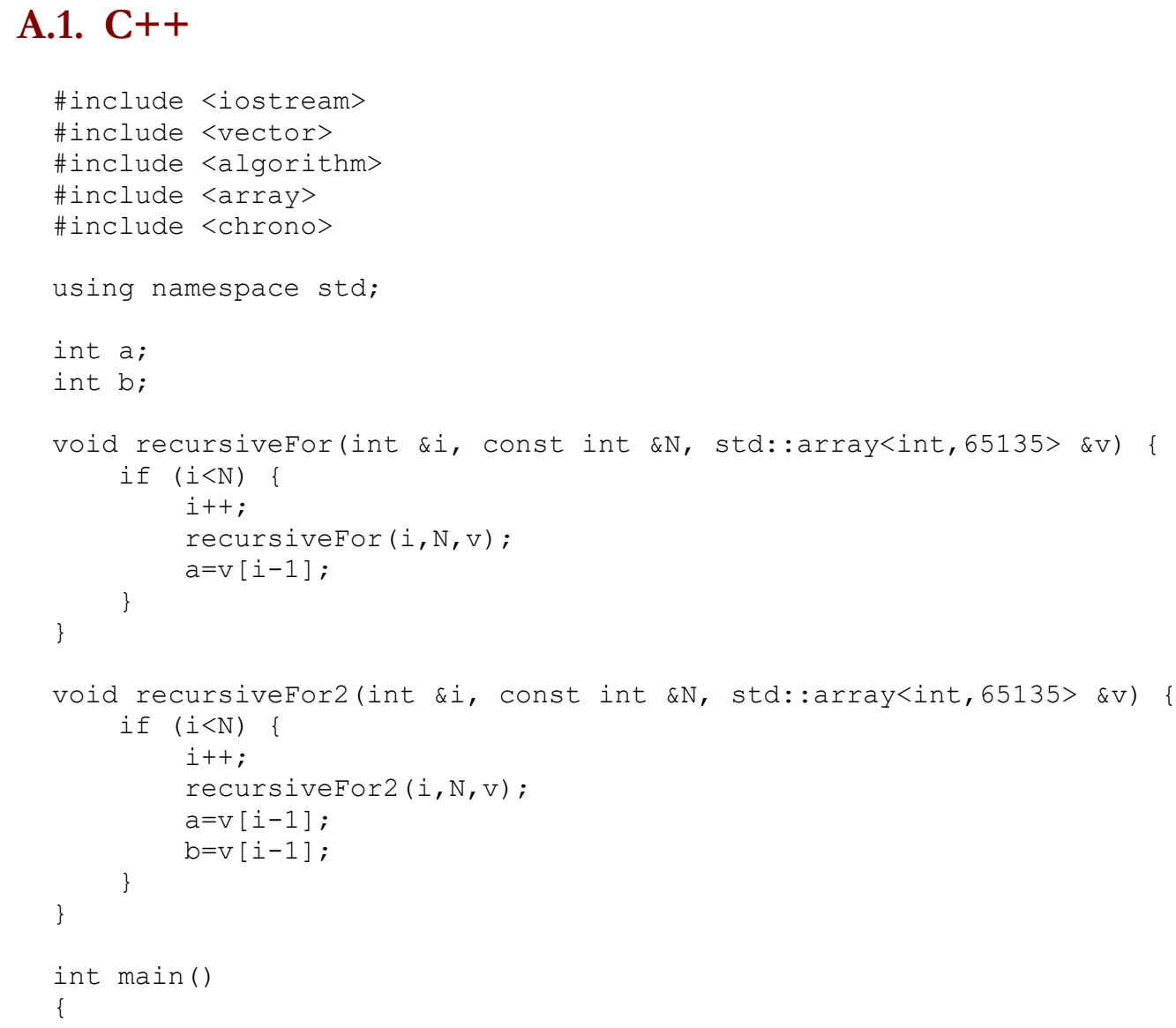




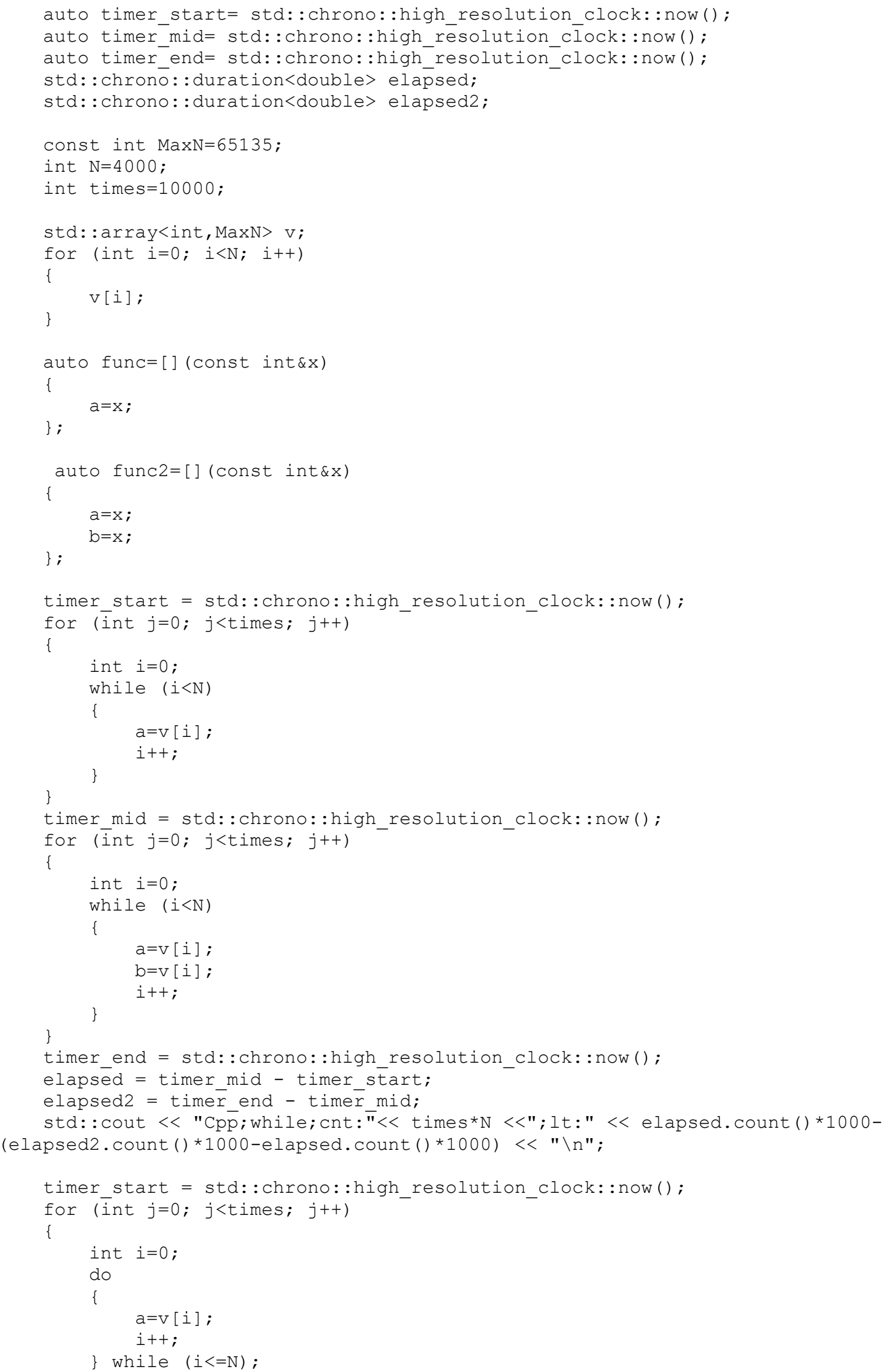

Central-European Journal of New Technologies in Research, Education and Practice 


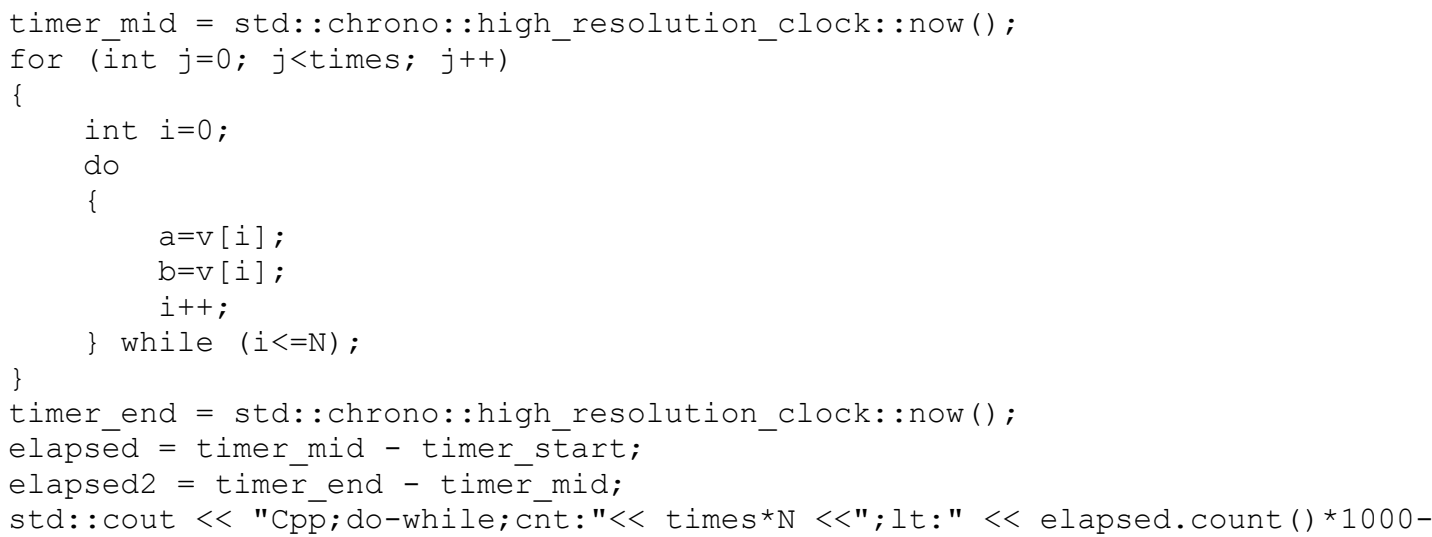

Central-European Journal of New Technologies in Research, Education and Practice 


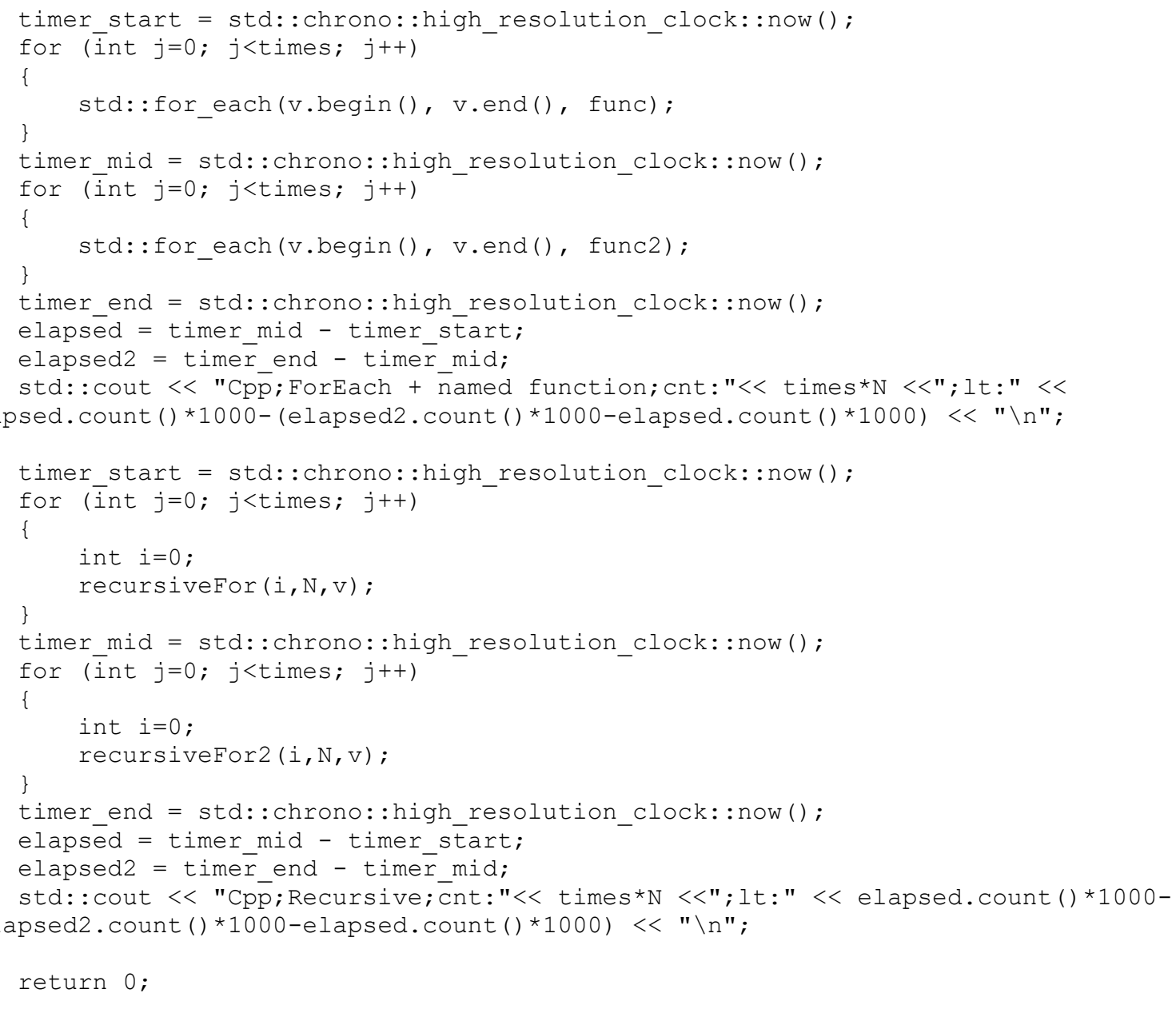

\section{A.2. C\#}

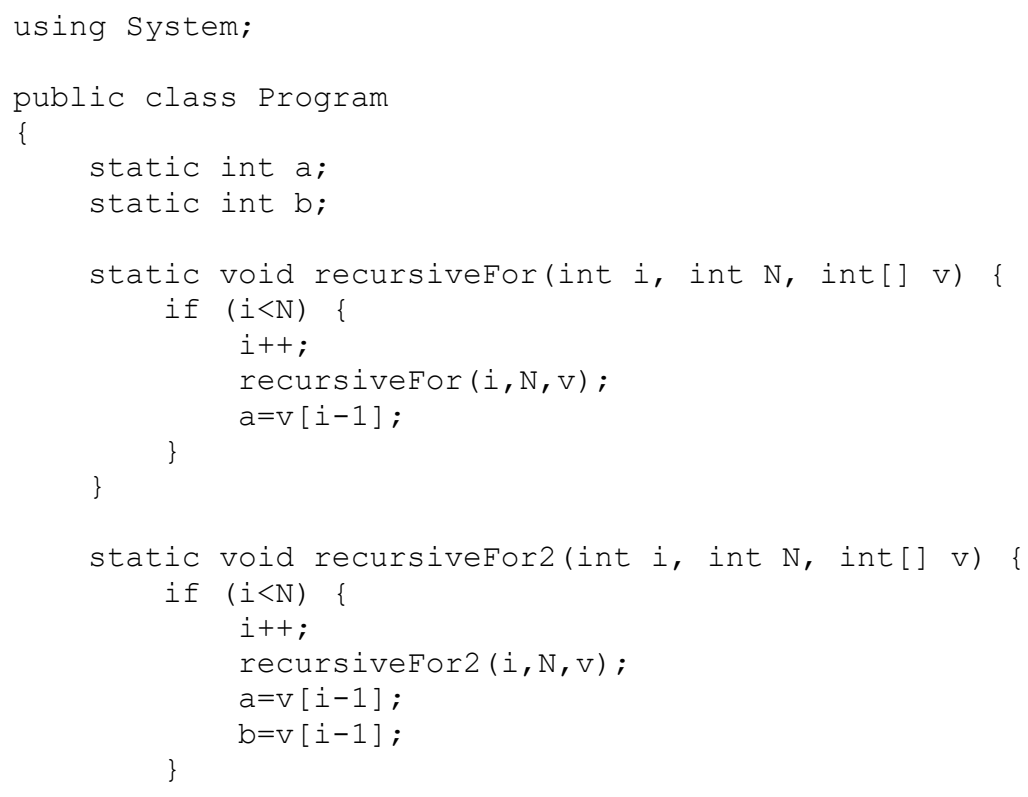

Central-European Journal of New Technologies in Research, Education and Practice 
\}

public static void Main()

\{

DateTime timer_start= DateTime.Now;

DateTime timer mid= DateTime.Now;

DateTime timer_end;

TimeSpan elapsed;

TimeSpan elapsed2;

int $\mathrm{N}=4000$;

int times $=10000$;

int [] $\mathrm{v}=$ new int $[\mathrm{N}]$;

for (int $i=0 ; i<N ; i++$ )

\{

$\mathrm{V}[\mathrm{i}]=\mathrm{i}$;

\}

Action<int $>$ func $=x=>\{$

$\mathrm{a}=\mathrm{x}$;

\} ;

Action<int $>$ func $2=x=>\{$

$a=x$;

$\mathrm{b}=\mathrm{x}$;

\} ;

timer start $=$ DateTime.Now;

for (int $j=0 ; j<t i m e s ; j++$ )

for (int $i=0 ; i<N ; i++)$

$\mathrm{a}=\mathrm{v}[\mathrm{i}]$;

timer mid= DateTime.Now

for (int $j=0 ; j<t i m e s ; j++$ )

\{

for (int $i=0 ; i<\mathrm{N} ; i++)$

\{

$\mathrm{a}=\mathrm{v}[\mathrm{i}]$

$\mathrm{b}=\mathrm{v}[\mathrm{i}]$;

\}

timer end = DateTime. Now;

elapsē = timer mid - timer start;

elapsed2 = timer end - timer mid;

Console.WriteLine ("cs; for; cnt: $\{1\} ; l t:\{0\}$ ", elapsed.Milliseconds-

(elapsed2.Milliseconds-elapsed.Milliseconds), $N^{*}$ times) ;

timer_start= DateTime.Now;

for (int $j=0 ; j<$ times; $j++$ )

\{

int $i=0$

while $(i<N)$

\{

$\mathrm{a}=\mathrm{v}[\mathrm{i}]$

$i++;$

\}

timer mid= DateTime.Now;

for (int $j=0 ; j<$ times; $j++$ )

int $i=0$;

Central-European Journal of New Technologies in Research, Education and Practice

Volume 2, Number 1, 2020. 


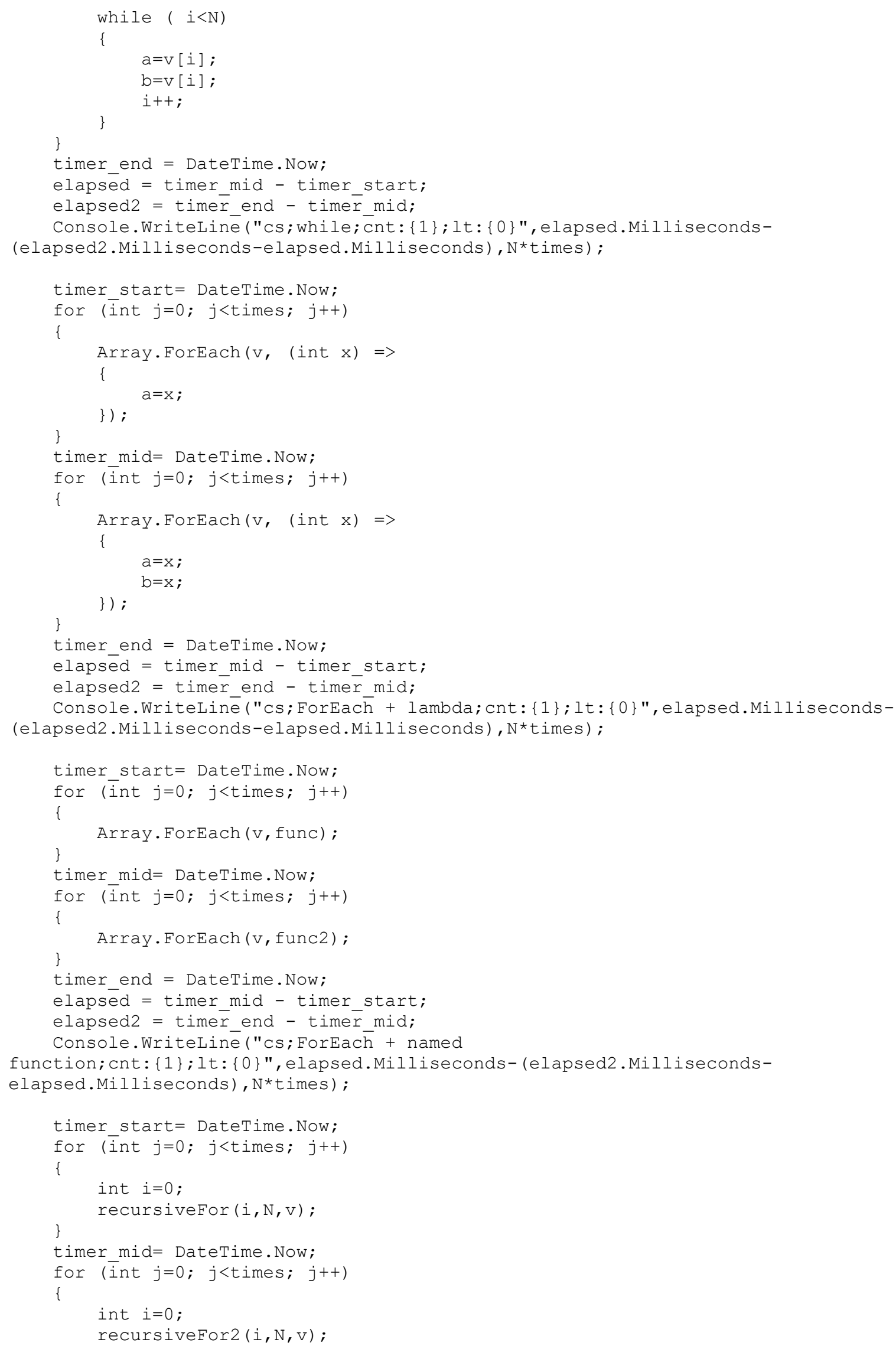

Central-European Journal of New Technologies in Research, Education and Practice 
\}

timer end = DateTime.Now;

elapsed = timer mid - timer_start;

elapsed2 = time $\bar{r}$ end - time $\bar{r}$ mid;

Console.WriteLinē ("cs; Recursīve; cnt: $\{1\}$; lt: $\{0\}$ ", elapsed.Milliseconds(elapsed2.Milliseconds-elapsed.Milliseconds), N*times);

\section{A.3. Java}

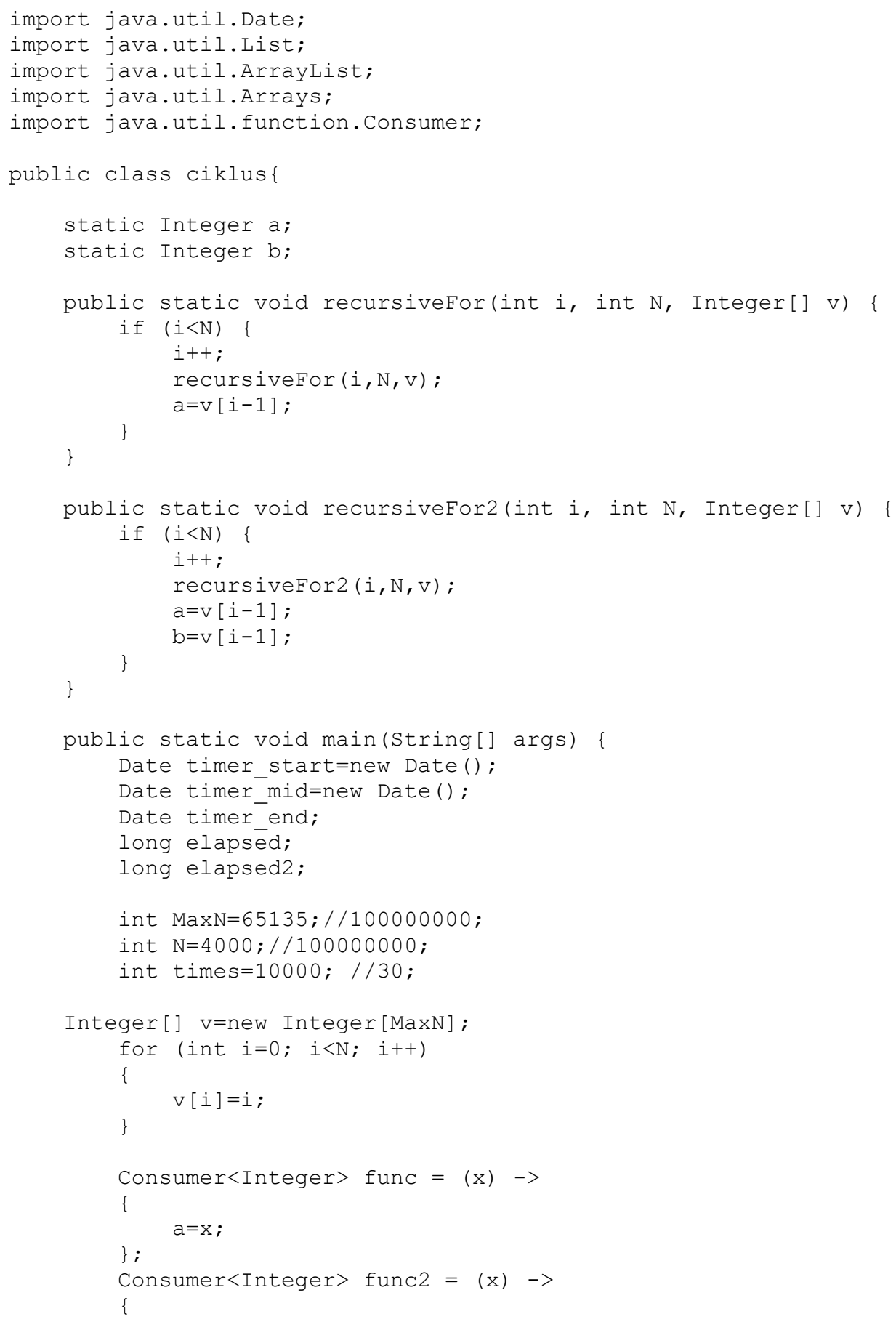

Central-European Journal of New Technologies in Research, Education and Practice 


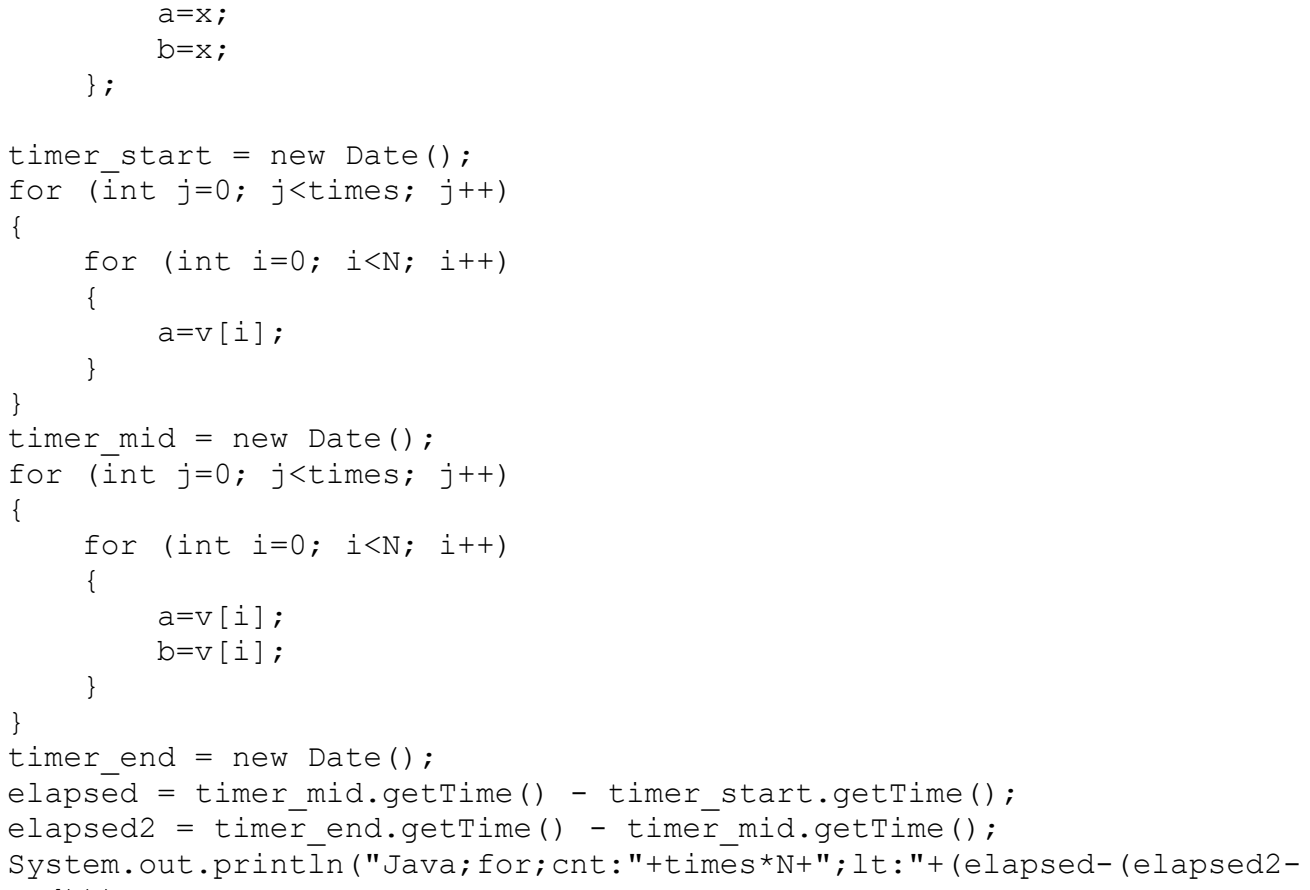

Central-European Journal of New Technologies in Research, Education and Practice 


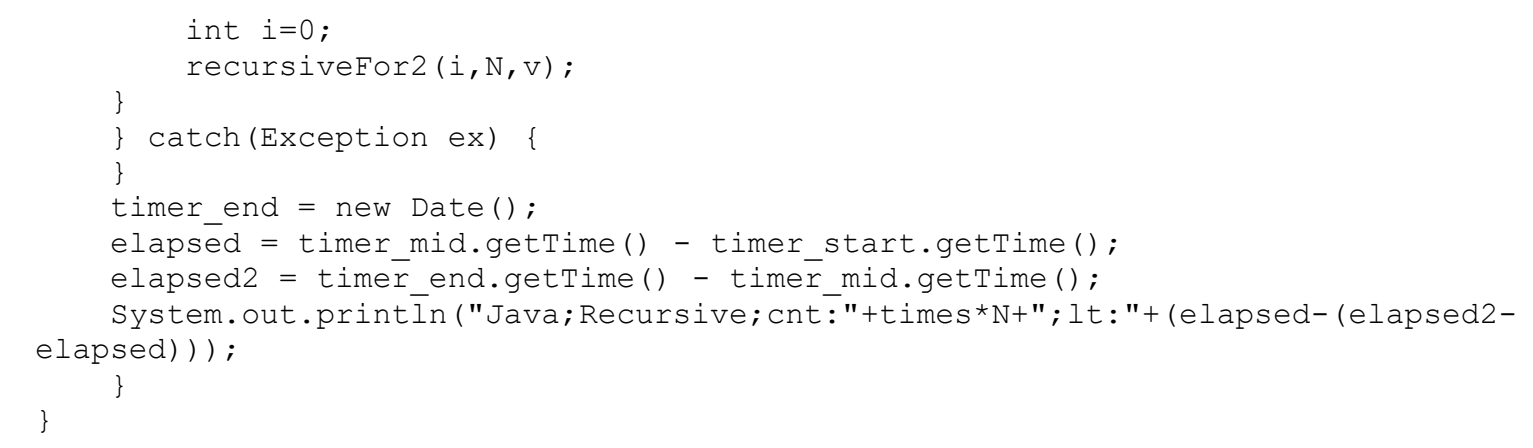

\section{A.4. JavaScript}

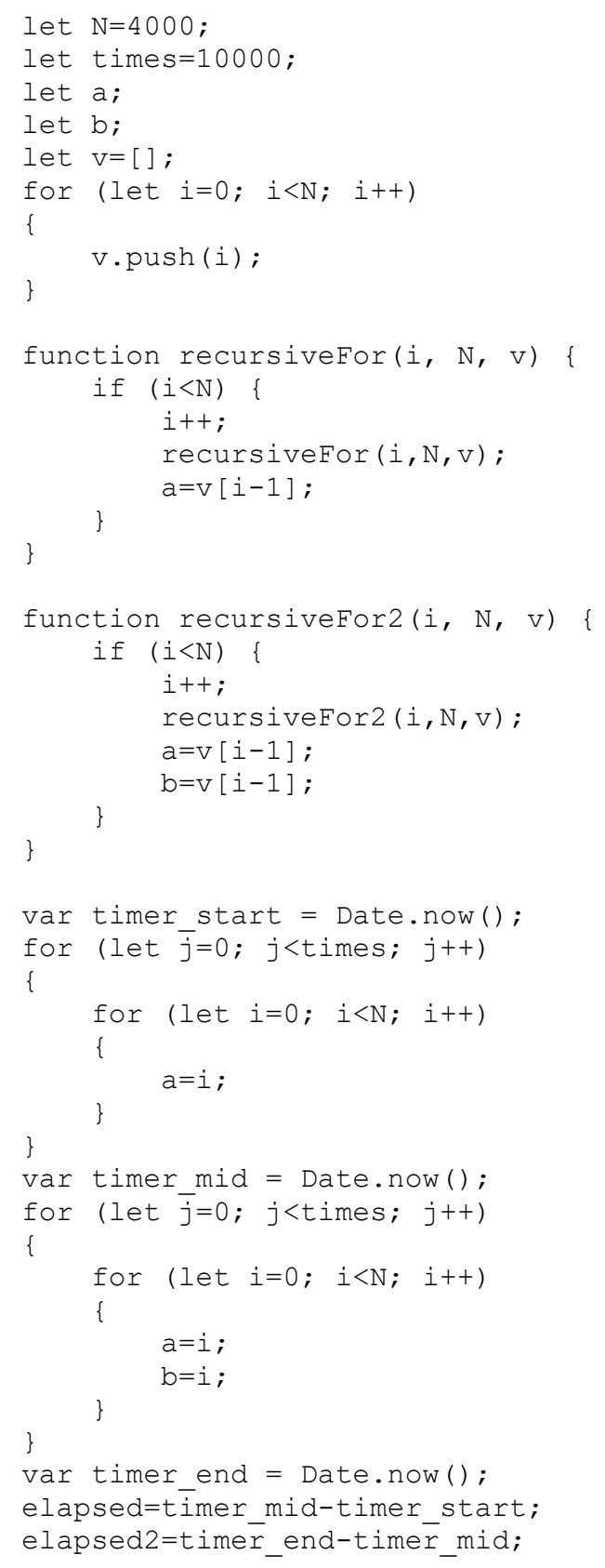

Central-European Journal of New Technologies in Research, Education and Practice 


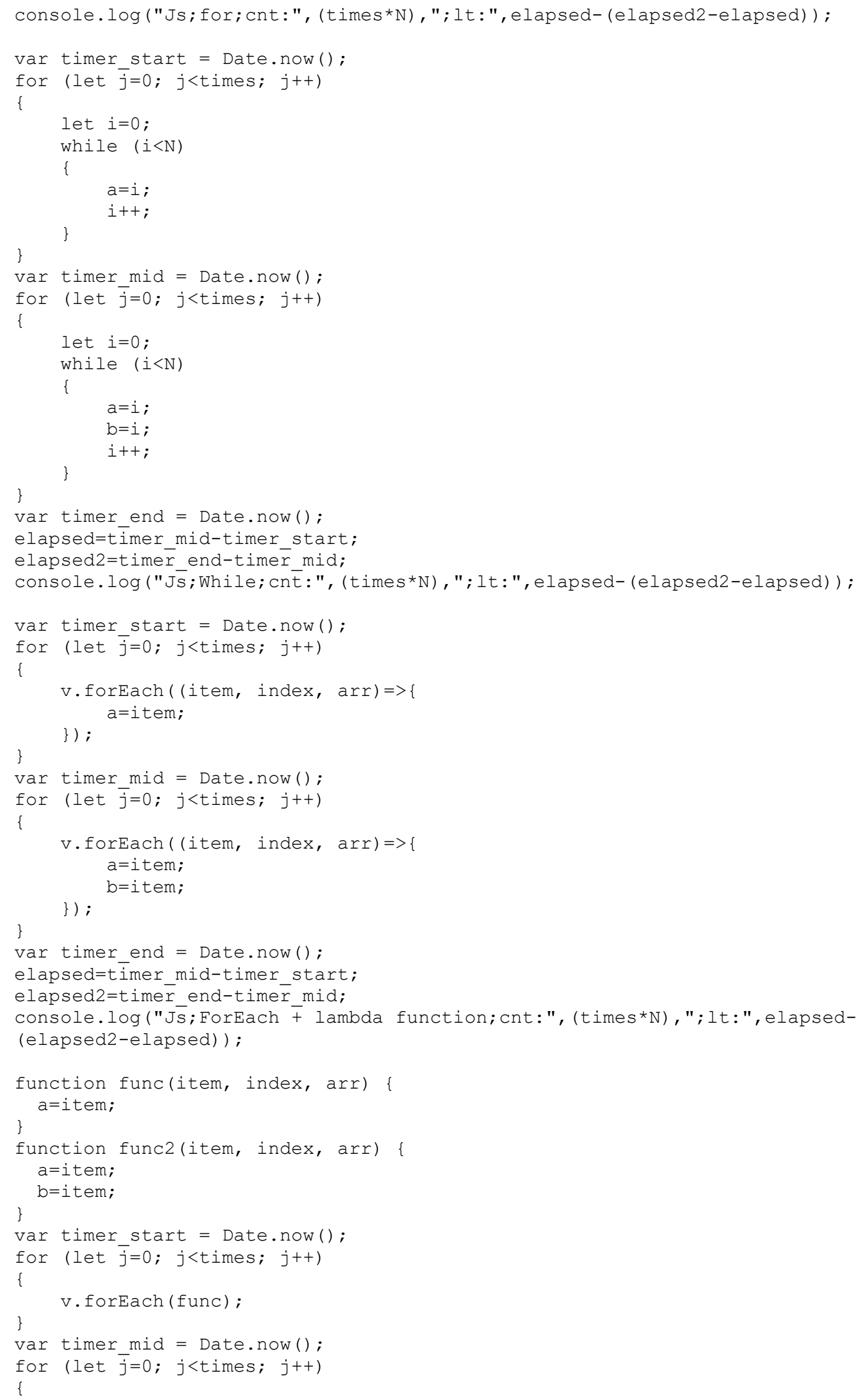

Central-European Journal of New Technologies in Research, Education and Practice 


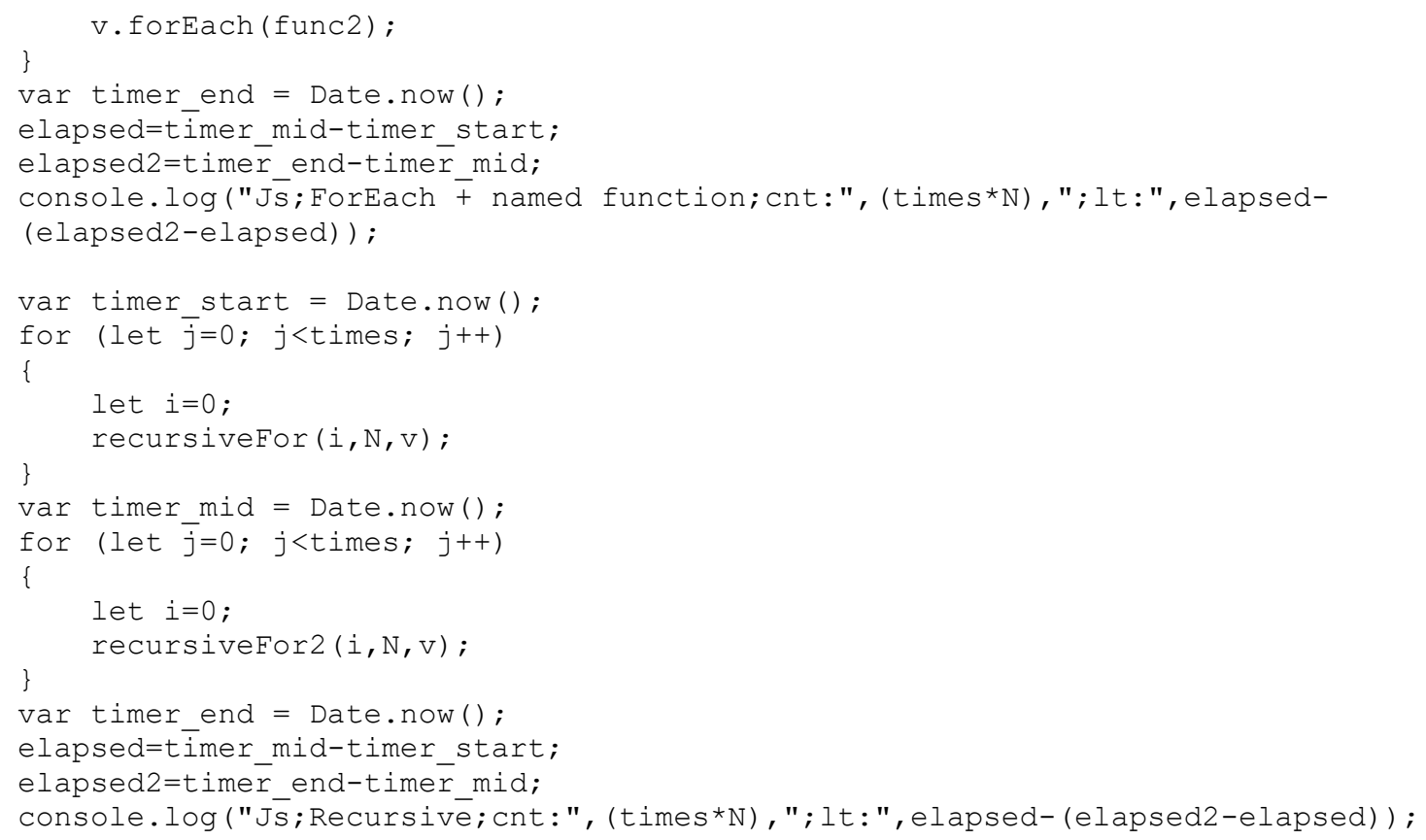

\section{A.5. Pascal}

Program ciklus;

Uses sysutils;

var

timer_start:TDateTime;

timer mid:TDateTime;

timer_end:TDateTime;

elapsed:TDateTime;

elapsed2: TDateTime;

$\mathrm{N}$ : integer;

times: integer;

v:array[1.65135] of integer;

i:integer;

$j$ :integer;

a:integer;

b: integer;

w: integer;

procedure recursiveFor(var i:integer; const $\mathrm{N}$ :integer; var v:array of integer); begin

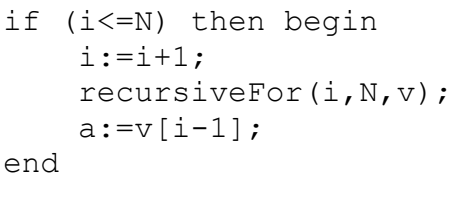

procedure recursiveFor2 (var i:integer; const N:integer; var v:array of integer); begin

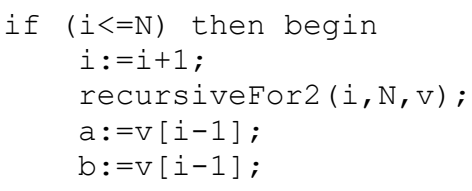

Central-European Journal of New Technologies in Research, Education and Practice

Volume 2, Number 1, 2020. 


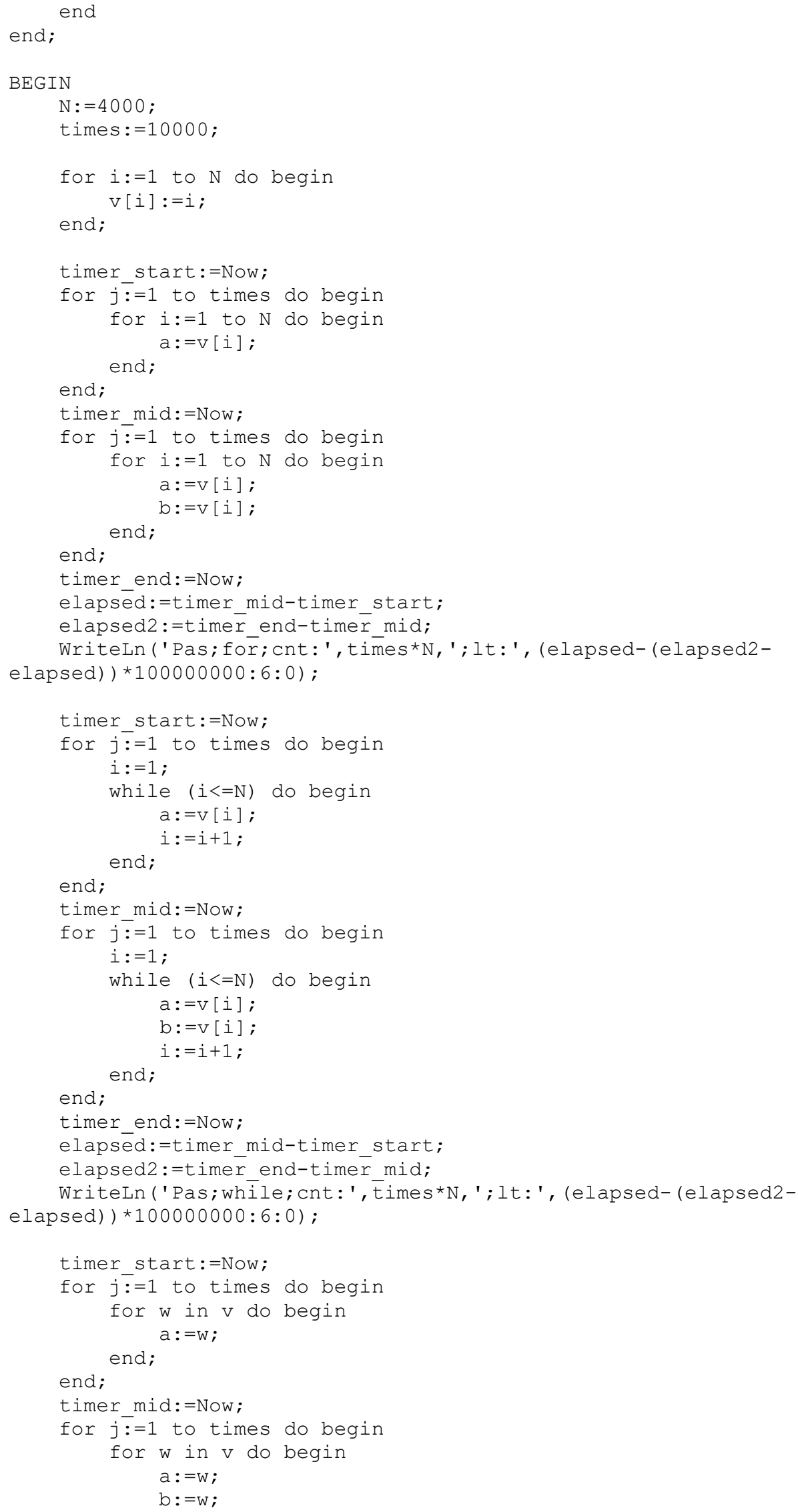

Central-European Journal of New Technologies in Research, Education and Practice 


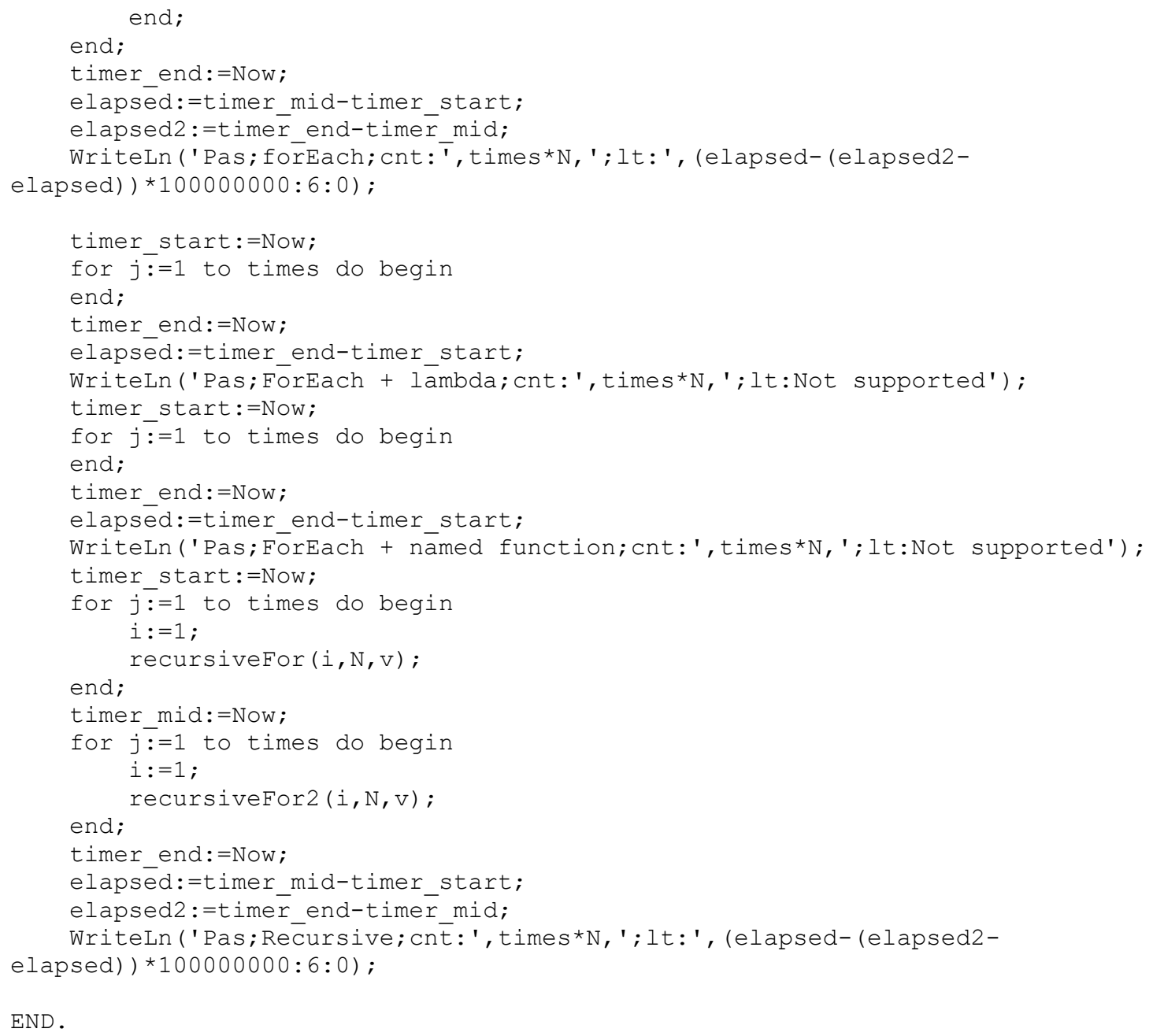

\section{A.6. Python}

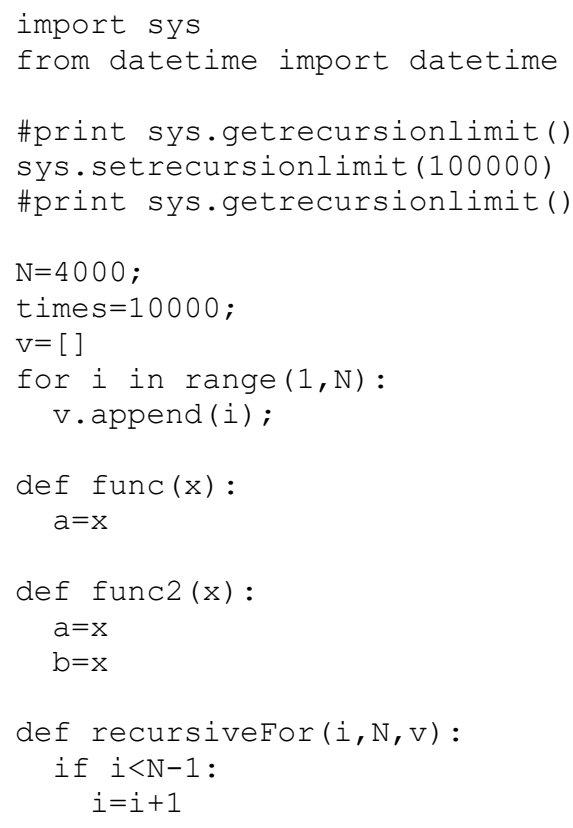

Central-European Journal of New Technologies in Research, Education and Practice 


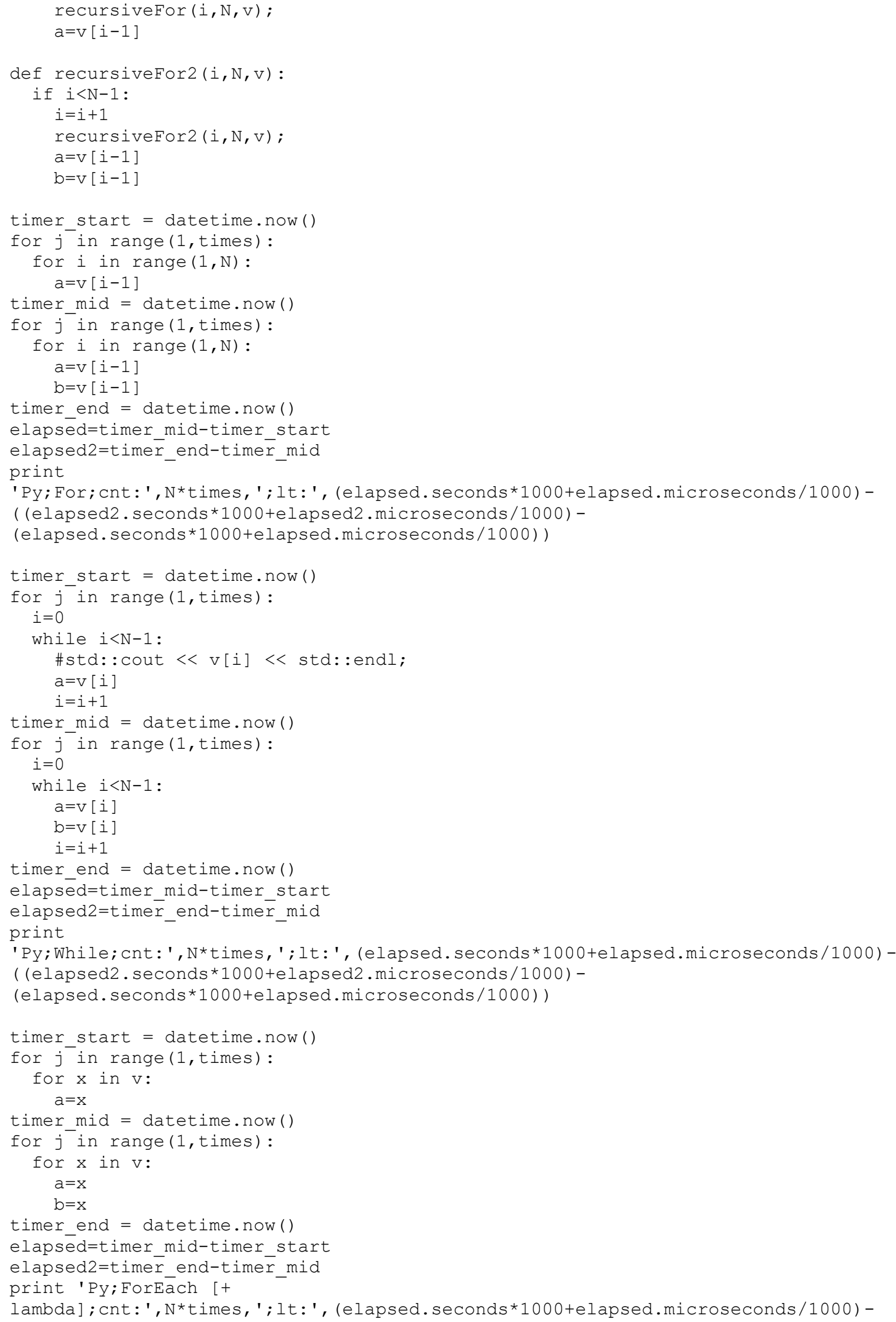

Central-European Journal of New Technologies in Research, Education and Practice 


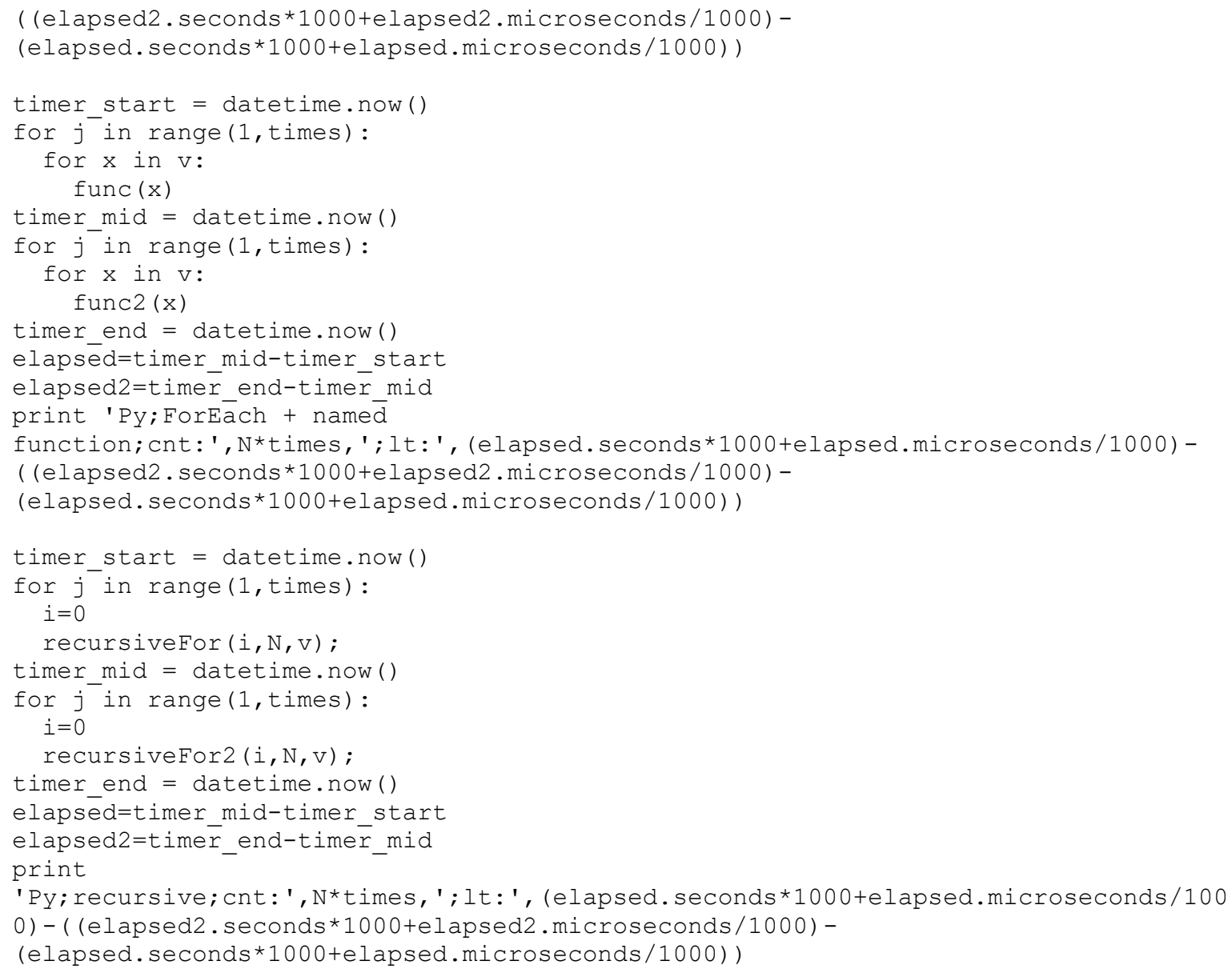

\section{B. Raw data of measurement}

Cpp; while; cnt:40000000; lt:0.056

Cpp; do-while; cnt: $40000000 ; 1 t: 0.658$

Cpp; for; cnt: $40000000 ; 1 t: 31.361$

Cpp; ForEach + lambda; cnt:40000000;1t:1076.23

Cpp; ForEach + named function; cnt:40000000;lt:1087.14

Cpp; Recursive; cnt: 40000000 ; lt: 124.673

Cs; for; cnt: $40000000 ; 1 t: 22$

cs; while; cnt:40000000; lt:19

CS; ForEach + lambda; cnt:40000000;lt:92

CS;ForEach + named function; cnt:40000000;1t:89

cs; Recursive; cnt: $40000000 ;$ it: 85

Java;for; cnt: $40000000 ; 1 t: 56$

Java; while; cnt:40000000; lt:51

Java; forEach; cnt: $40000000 ; 1 t: 213$

Java; ForEach + lambda; cnt:40000000;lt:153

Java; ForEach + named function; cnt:40000000;lt:1901

Java; Recursive; cnt: $40000000 ; 1 t: 98$

Js; for;cnt: 40000000 ; lt: 36

Js; While; cnt: 40000000 ; it: 34

Js; ForEach + lambda function; cnt: 40000000 ;lt: 249

Js;ForEach + named function; cnt: 40000000 ; lt: 229

Js; Recursive; cnt: 40000000 ; lt: 263

Pas;for;cnt:40000000; lt: 97

Pas; while; cnt:40000000;lt: 58

Pas; forEach; cnt: 40000000 ; lt: 549

Central-European Journal of New Technologies in Research, Education and Practice

Volume 2, Number 1, 2020. 
Pas;ForEach + lambda; cnt:40000000;lt: Not supported

Pas;ForEach + named function; cnt:40000000;lt:Not supported

Pas; Recursive; cnt:40000000;lt: 130

Py;For; cnt: 40000000 ; lt: 1140

Py; While; cnt: 40000000 ;lt: 1872

Py; ForEach [+ lambda]; cnt: 40000000 ; lt: 265

Py; ForEach + named function; cnt: 40000000 ; lt: 3176

Py; recursive; cnt: 40000000 ; lt: 9022

Cpp; while; cnt: 40000000 ; lt: 11.553

Cpp; do-while; cnt: $40000000 ;$ it:0.589

Cpp; for; cnt:40000000; lt:22.609

Cpp; ForEach + lambda; cnt:40000000;lt:1095.92

Cpp;ForEach + named function; cnt:40000000;lt:1125.63

Cpp; Recursive; cnt: 40000000 ; lt:110.243

cs; for; cnt: $40000000 ; 1 t: 31$

cs; while; cnt: $40000000 ; 1 t: 8$

CS; ForEach + lambda; cnt:40000000;lt:117

CS; ForEach + named function; cnt:40000000;lt:92

cs; Recursive; cnt: $40000000 ;$ it: 91

Java; for; cnt: $40000000 ; 1 t: 37$

Java; while; cnt: $40000000 ; 1 t: 48$

Java; forEach; cnt:40000000; lt:199

Java; ForEach + lambda; cnt:40000000; lt:157

Java;ForEach + named function; cnt:40000000;lt:1961

Java; Recursive; cnt: $40000000 ; 1 t: 138$

Js; for; cnt: 40000000 ; lt: 37

Js; While; cnt: 40000000 ; lt: 30

Js;ForEach + lambda function; cnt: 40000000 ;lt: 235

Js;ForEach + named function; cnt: 40000000 ;lt: 227

Js; Recursive; cnt: 40000000 ; lt: 251

Pas;for;cnt:40000000; lt: 101

Pas; while; cnt:40000000;lt: 94

Pas; forEach; cnt:40000000; lt: 546

Pas;ForEach + lambda; cnt:40000000;lt: Not supported

Pas;ForEach + named function; cnt:40000000;lt: Not supported

Pas; Recursive; cnt:40000000; lt: 118

Py; For; cnt: 40000000 ; lt: 1204

Py; While; cnt: 40000000 ; it: 2665

Py; ForEach [+ lambda]; cnt: 40000000 ; lt: 270

Py;ForEach + named function; cnt: 40000000 ; lt: 3197

Py; recursive; cnt: 40000000 ; lt: 8994

Cpp; while; cnt:40000000; lt:24.209

Cpp; do-while; cnt:40000000; lt:1.789

Cpp; for; cnt: $40000000 ; 1 t: 4.774$

Cpp; ForEach + lambda; cnt:40000000;1t:1095

Cpp;ForEach + named function; cnt:40000000;1t:1109.37

Cpp; Recursive; cnt: $40000000 ; 1 t: 126.22$

cs; for; cnt: $40000000 ; 1 t: 47$

cs; while; cnt: $40000000 ; 1 t: 2$

Cs; ForEach + lambda; cnt:40000000;lt:90

CS; ForEach + named function; cnt:40000000;lt:92

cs; Recursive; cnt: 40000000 ; lt: 93

Java; for; cnt:40000000; lt:50

Java; while; cnt: $40000000 ;$ it: 66

Java; forEach; cnt: $40000000 ; 1 t: 228$

Java; ForEach + lambda; cnt:40000000;lt:157

Java; ForEach + named function; cnt:40000000; lt:1938

Java; Recursive; cnt:40000000; lt:112

Js; for; cnt: 40000000 ; lt: 37

Js; While; cnt: 40000000 ; lt: 33

Js; ForEach + lambda function; cnt: 40000000 ; lt: 238

Js; ForEach + named function; cnt: 40000000 ; lt: 231

Central-European Journal of New Technologies in Research, Education and Practice

Volume 2, Number 1, 2020. 


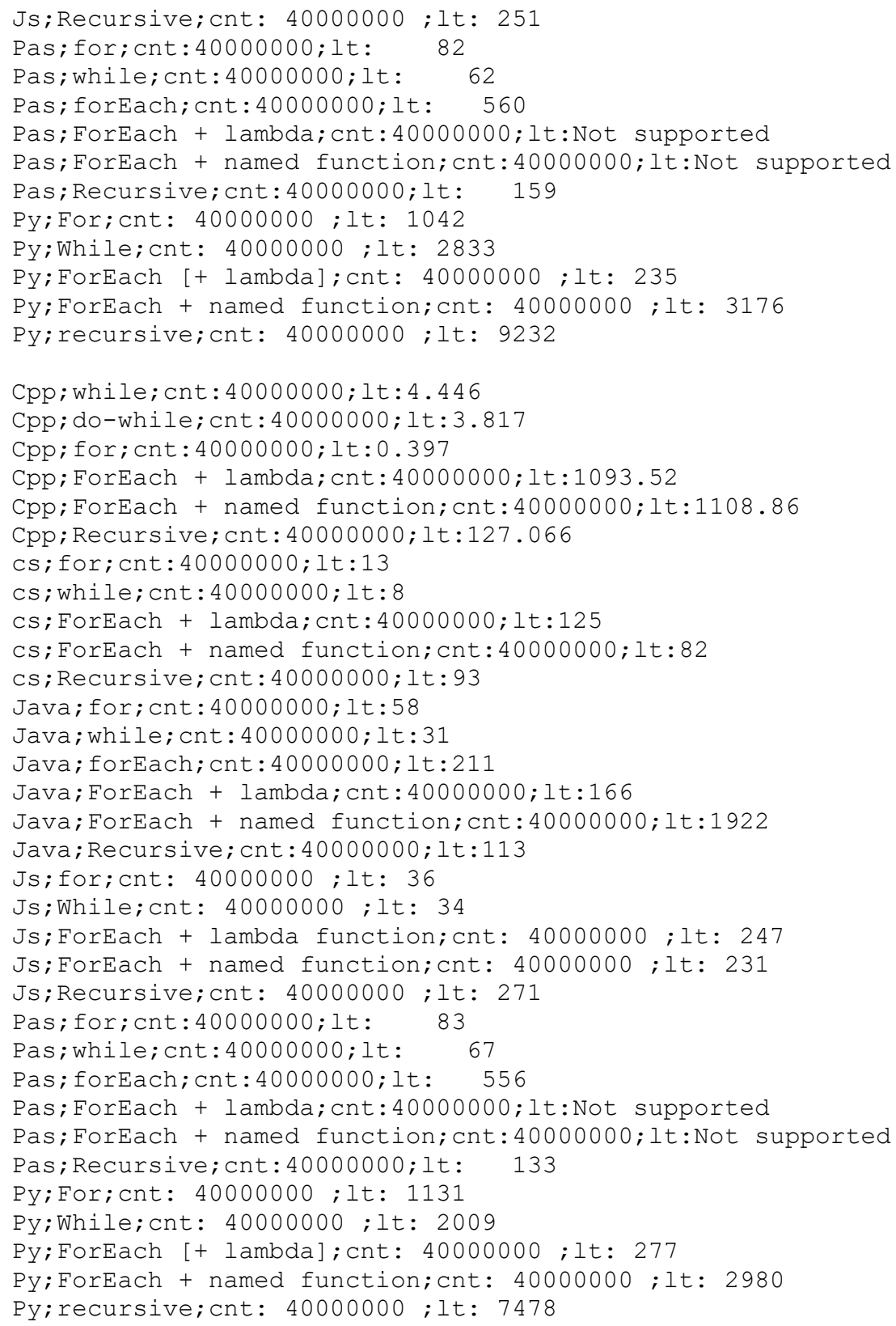

Cpp; while; cnt:40000000; lt:3.886

Cpp; do-while; cnt: $40000000 ; 1 t: 0.068$

Cpp; for; cnt: $40000000 ; 1 t: 2.29$

Cpp; ForEach + lambda; cnt:40000000;1t:1114.82

Cpp; ForEach + named function; cnt:40000000;lt:1160.19

Cpp; Recursive; cnt: 40000000 ; lt:134.357

cs; for; cnt: 40000000 ; lt: 20

cs; while; cnt: $40000000 ; 1 t: 21$

CS; ForEach + lambda; cnt:40000000;lt:91

CS; ForEach + named function; cnt:40000000;lt:93

cs; Recursive; cnt: $40000000 ;$ lt: 86

Java;for; cnt: $40000000 ; 1 t: 65$

Java; while; cnt:40000000;lt: 61

Java; forEach; cnt: $40000000 ; 1 t: 222$

Java; ForEach + lambda; cnt:40000000; lt:160

Java; ForEach + named function; cnt:40000000;lt:2018

Java; Recursive; cnt:40000000; lt:117

Central-European Journal of New Technologies in Research, Education and Practice 
Js; for; cnt: 40000000 ; lt: 36

Js; While; cnt: 40000000 ; lt: 34

Js;ForEach + lambda function; cnt: 40000000 ;lt: 252

Js;ForEach + named function; cnt: 40000000 ; lt: 239

Js; Recursive; cnt: 40000000 ; lt: 270

Pas;for;cnt:40000000;lt: 83

Pas; while; cnt: 40000000 ; lt: 67

Pas;forEach; cnt: 40000000 ; lt: 564

Pas;ForEach + lambda; cnt:40000000;lt:Not supported

Pas;ForEach + named function; cnt:40000000; lt: Not supported

Pas; Recursive; cnt:40000000; lt: 137

Py;For; cnt: 40000000 ; lt: 1190

Py; While; cnt: 40000000 ;lt: 1832

Py; ForEach [+ lambda]; cnt: 40000000 ; lt: 300

Py; ForEach + named function; cnt: 40000000 ; lt: 3291

Py; recursive; cnt: 40000000 ; lt: 9734

Cpp; while; cnt:40000000; lt:5.952

Cpp; do-while; cnt:40000000;lt:3.779

Cpp; for; cnt: $40000000 ; 1 t: 5.6$

Cpp;ForEach + lambda; cnt:40000000;1t:1136.82

Cpp; ForEach + named function; cnt:40000000; lt:1141.19

Cpp; Recursive; cnt: $40000000 ; 1 t: 133.593$

cs; for; cnt: $40000000 ; 1 t: 23$

cs; while; cnt: $40000000 ; 1 t: 22$

Cs;ForEach + lambda; cnt:40000000;lt:93

CS; ForEach + named function; cnt:40000000;lt:91

cs; Recursive; cnt: $40000000 ;$ lt: 89

Java; for; cnt: $40000000 ; 1 t: 38$

Java; while; cnt: $40000000 ;$ lt: 54

Java; forEach; cnt: $40000000 ; 1 t: 218$

Java; ForEach + lambda; cnt:40000000;lt:177

Java;ForEach + named function; cnt:40000000; lt:2018

Java; Recursive; cnt: $40000000 ; 1 t: 110$

Js; for; cnt: 40000000 ; lt: 40

Js; While; cnt: 40000000 ; lt: 40

Js; ForEach + lambda function; cnt: 40000000 ; lt: 253

Js; ForEach + named function; cnt: 40000000 ; lt: 239

Js; Recursive; cnt: 40000000 ; lt: 265

Pas;for;cnt:40000000;lt: 81

Pas; while; cnt:40000000;lt: 66

Pas; forEach; cnt:40000000; lt: 565

Pas;ForEach + lambda; cnt:40000000;lt:Not supported

Pas;ForEach + named function; cnt:40000000;lt: Not supported

Pas; Recursive; cnt:40000000; lt: 131

Py; For; cnt: 40000000 ; lt: 101

Py; While; cnt: 40000000 ; lt: 1431

Py; ForEach [+ lambda]; cnt: 40000000 ; lt: 1225

Py; ForEach + named function; cnt: 40000000 ; lt: 5895

Py; recursive; cnt: 40000000 ; lt: 14090

Cpp; while; cnt: $40000000 ; 1 t: 5.244$

Cpp; do-while; cnt: $40000000 ; 1 t: 0.978$

Cpp; for; cnt: $40000000 ; 1 t: 3.862$

Cpp; ForEach + lambda; cnt:40000000;1t:1139.48

Cpp;ForEach + named function; cnt:40000000;1t:1143.29

Cpp; Recursive; cnt: 40000000 ; lt:133.426

Cs; for; cnt: $40000000 ; 1 t: 23$

cs; while; cnt: 40000000 ; lt: 21

Cs;ForEach + lambda; cnt:40000000;lt:93

CS; ForEach + named function; cnt:40000000;lt:94

cs; Recursive; cnt: $40000000 ; 1 t: 89$

Java; for; cnt: $40000000 ; 1 t: 38$

Java; while; cnt: $40000000 ;$ it: 48

Central-European Journal of New Technologies in Research, Education and Practice 
Java; forEach; cnt: $40000000 ; 1 t: 232$

Java;ForEach + lambda; cnt:40000000; lt:172

Java; ForEach + named function; cnt:40000000;lt:2012

Java; Recursive; cnt: 40000000 ; lt:114

Js;for; cnt: 40000000 ; lt: 36

Js; While; cnt: 40000000 ; lt: 36

Js;ForEach + lambda function; cnt: 40000000 ; lt: 247

Js;ForEach + named function; cnt: 40000000 ; lt: 237

Js; Recursive; cnt: 40000000 ; lt: 272

Pas;for;cnt:40000000; lt: 83

Pas; while; cnt:40000000; lt: 67

Pas; forEach; cnt:40000000; lt: 565

Pas;ForEach + lambda; cnt:40000000;lt:Not supported

Pas;ForEach + named function; cnt:40000000;lt: Not supported

Pas;Recursive; cnt:40000000; it: 139

Py;For; cnt: 40000000 ; lt: 1654

Py; While; cnt: 40000000 ; lt: 2797

Py; ForEach [+ lambda]; cnt: 40000000 ; lt: 265

Py; ForEach + named function; cnt: 40000000 ; lt: 3279

Py; recursive; cnt: 40000000 ; lt: 10467

Cpp; while; cnt: $40000000 ; 1 t: 5.164$

Cpp; do-while; cnt:40000000;lt:2.288

Cpp; for; cnt: $40000000 ; 1 t: 4.173$

Cpp; ForEach + lambda; cnt:40000000;lt:1149.63

Cpp; ForEach + named function; cnt:40000000;lt:1146.72

Cpp; Recursive; cnt: $40000000 ; 1 t: 134.877$

cs; for; cnt: $40000000 ; 1 t: 24$

cs; while; cnt: $40000000 ; 1 t: 22$

cs; ForEach + lambda; cnt:40000000;lt:93

CS; ForEach + named function; cnt:40000000;1t:91

cs; Recursive; cnt:40000000; 1t:111

Java; for; cnt:40000000; lt:59

Java; while; cnt:40000000;lt:52

Java; forEach; cnt: $40000000 ; 1 t: 247$

Java; ForEach + lambda; cnt:40000000;lt:107

Java; ForEach + named function; cnt:40000000; lt:2035

Java; Recursive; cnt: $40000000 ;$ it: 114

Js; for; cnt: 40000000 ; lt: 36

Js; While; cnt: 40000000 ; lt: 34

Js; ForEach + lambda function; cnt: 40000000 ;lt: 259

Js;ForEach + named function; cnt: 40000000 ; lt: 244

Js; Recursive; cnt: 40000000 ; lt: 280

Pas;for;cnt:40000000;lt: 83

Pas; while; cnt:40000000;it: 65

Pas; forEach; cnt:40000000;it: 646

Pas; ForEach + lambda; cnt:40000000;lt:Not supported

Pas;ForEach + named function; cnt:40000000; lt: Not supported

Pas; Recursive; cnt:40000000; lt: 152

Py;For; cnt: 40000000 ; lt: 1313

Py; While; cnt: 40000000 ; lt: 1905

Py; ForEach [+ lambda]; cnt: $40000000 ; 1 t: 268$

Py; ForEach + named function; cnt: 40000000 ; lt: 3216

Py; recursive; cnt: 40000000 ; lt: 9942

Cpp; while; cnt:40000000; lt:0.999

Cpp; do-while; cnt:40000000; lt:2.048

Cpp; for; cnt: $40000000 ; 1 t: 0.98$

Cpp;ForEach + lambda; cnt:40000000;1t:1216.67

Cpp; ForEach + named function; cnt:40000000;lt:1147.93

Cpp; Recursive; cnt:40000000; lt:139.67

cs; for; cnt: $40000000 ; 1 t: 23$

cs; while; cnt: 40000000 ; 1 t: 21

CS; ForEach + lambda; cnt:40000000; lt:95

Central-European Journal of New Technologies in Research, Education and Practice

Volume 2, Number 1, 2020. 


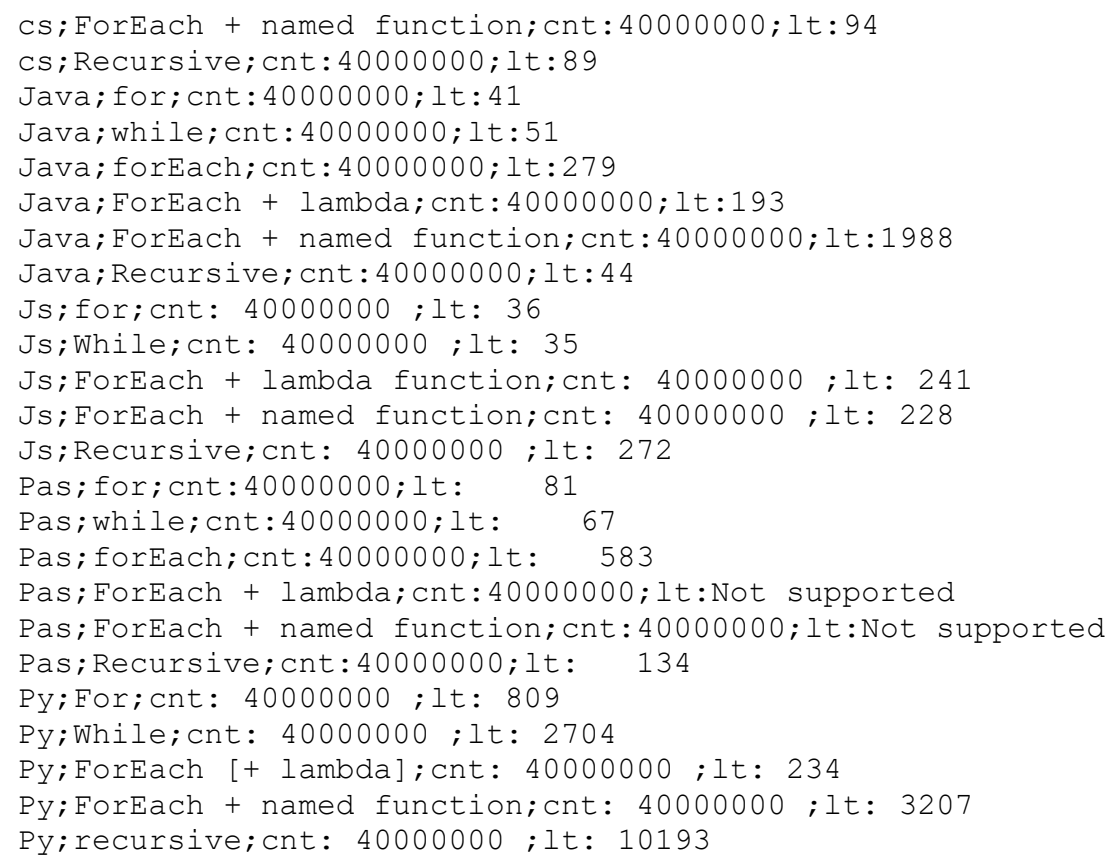

Cpp; while; cnt:40000000; lt: 1.675

Cpp; do-while; cnt: $40000000 ; 1 t: 1.435$

Cpp; for; cnt: $40000000 ; 1 t: 4.495$

Cpp; ForEach + lambda; cnt:40000000;1t:1117.01

Cpp;ForEach + named function; cnt:40000000;1t:1149.84

Cpp; Recursive; cnt: $40000000 ;$ lt:127.438

CS; for; cnt: $40000000 ;$ it: 21

cs; while; cnt: $40000000 ; 1 t: 7$

Cs; ForEach + lambda; cnt:40000000;1t:118

CS; ForEach + named function; cnt:40000000;lt:92

cs; Recursive; cnt: $40000000 ; 1 t: 86$

Java;for; cnt:40000000; lt: 57

Java; while; cnt: $40000000 ;$ it: 47

Java; forEach; cnt: $40000000 ; 1 t: 228$

Java; ForEach + lambda; cnt:40000000;lt:175

Java;ForEach + named function; cnt:40000000;1t:1965

Java; Recursive; cnt:40000000; lt:117

Js;for; cnt: 40000000 ; lt: 36

Js; While; cnt: 40000000 ; lt: 33

Js;ForEach + lambda function; cnt: 40000000 ; lt: 244

Js;ForEach + named function; cnt: 40000000 ; lt: 218

Js; Recursive; cnt: 40000000 ; lt: 259

Pas;for;cnt:40000000;lt: 82

Pas; while; cnt:40000000; it: 64

Pas;forEach; cnt:40000000; lt: 544

Pas; ForEach + lambda; cnt:40000000;lt: Not supported

Pas;ForEach + named function; cnt:40000000;lt:Not supported

Pas;Recursive; cnt:40000000;lt: 132

Py; For; cnt: 40000000 ; lt: 75

Py; While; cnt: 40000000 ; lt: 3586

Py; ForEach [+ lambda]; cnt: 40000000 ; lt: 975

Py; ForEach + named function; cnt: 40000000 ; lt: 3906

Py; recursive; cnt: 40000000 ; lt: 8930

Cpp; while; cnt: $40000000 ; 1 t: 48.477$

Cpp;do-while; cnt:40000000;lt:174.121

Cpp; for; cnt: $40000000 ; 1 t: 0.981$

Cpp; ForEach + lambda; cnt:40000000;lt:1697.38

Cpp; ForEach + named function; cnt:40000000;lt:1959.92

Central-European Journal of New Technologies in Research, Education and Practice 
Cpp; Recursive; cnt: $40000000 ; 1 t: 216.816$

Cs; for; cnt: $40000000 ; 1$ t: 88

cs; while; cnt: $40000000 ; 1 t: 60$

CS;ForEach + lambda; cnt:40000000;1t:311

CS; ForEach + named function; cnt:40000000;lt:212

cs; Recursive; cnt:40000000; 1t:128

Java; for; cnt: $40000000 ; 1 t: 76$

Java; while; cnt:40000000;lt:183

Java; forEach; cnt: 40000000 ; lt: 510

Java; ForEach + lambda; cnt:40000000;lt:324

Java; ForEach + named function; cnt:40000000;lt:3482

Java; Recursive; cnt: $40000000 ; 1 t: 148$

Js;for; cnt: 40000000 ; lt: 206

Js; While;cnt: 40000000 ;lt: 115

Js; ForEach + lambda function; cnt: 40000000 ;lt: 529

Js;ForEach + named function; cnt: 40000000 ; lt: 448

Js; Recursive; cnt: 40000000 ; lt: 400

Pas; for; cnt: 40000000 ; lt: 278

Pas; while; cnt: 40000000 ; lt: 60

Pas; forEach; cnt:40000000;lt: 817

Pas;ForEach + lambda; cnt:40000000;lt: Not supported

Pas;ForEach + named function; cnt:40000000;lt:Not supported

Pas; Recursive; cnt:40000000;lt: 215

Py; For; cnt: 40000000 ; lt: 1964

Py; While; cnt: 40000000 ; lt: 3432

Py; ForEach [+ lambda]; cnt: 40000000 ; lt: 1442

Py; ForEach + named function; cnt: 40000000 ; lt: 5341

Py; recursive; cnt: 40000000 ; lt: 14436

Cpp; while; cnt: 40000000 ; lt: 13.586

Cpp; do-while; cnt: $40000000 ; 1 t: 1.62$

Cpp; for; cnt: $40000000 ; 1 t: 5.154$

Cpp; ForEach + lambda; cnt:40000000;lt:1106.2

Cpp;ForEach + named function; cnt:40000000;lt:1108.6

Cpp; Recursive; cnt:40000000; lt:127.709

cs; for; cnt: $40000000 ; 1$ t: 14

cs; while; cnt: $40000000 ; 1 t: 31$

CS; ForEach + lambda; cnt:40000000;lt:113

CS; ForEach + named function; cnt:40000000;1t:88

cs; Recursive; cnt: $40000000 ; 1 t: 94$

Java;for; cnt: $40000000 ; 1 t: 34$

Java; while; cnt: $40000000 ; 1 t: 40$

Java; forEach; cnt: 40000000 ; lt:211

Java; ForEach + lambda; cnt:40000000; lt:154

Java; ForEach + named function; cnt:40000000;lt:1977

Java; Recursive; cnt:40000000; lt:110

Js;for; cnt: 40000000 ;lt: 34

Js; While; cnt: 40000000 ; lt: 35

Js;ForEach + lambda function; cnt: 40000000 ; lt: 249

Js;ForEach + named function; cnt: 40000000 ; lt: 249

Js; Recursive; cnt: 40000000 ; lt: 260

Pas; for; cnt: 40000000 ; lt: 81

Pas; while; cnt:40000000;lt: 69

Pas; forEach; cnt:40000000; it: 515

Pas;ForEach + lambda; cnt:40000000;lt: Not supported

Pas;ForEach + named function; cnt:40000000;lt: Not supported

Pas;Recursive; cnt:40000000; lt: 128

Py; For; cnt: 40000000 ; lt: 1158

Py; While; cnt: 40000000 ; it: 1598

Py; ForEach [+ lambda]; cnt: 40000000 ; lt: 735

Py; ForEach + named function; cnt: 40000000 ; lt: 3325

Py; recursive; cnt: 40000000 ; lt: 9003

Cpp; while; cnt:40000000; lt: 7.54

Central-European Journal of New Technologies in Research, Education and Practice

Volume 2, Number 1, 2020. 
Cpp;do-while; cnt:40000000;lt:6.197

Cpp; for; cnt: $40000000 ; 1 t: 0.663$

Cpp; ForEach + lambda; cnt:40000000;lt:1136.38

Cpp;ForEach + named function; cnt:40000000;lt:1125.48

Cpp; Recursive; cnt: $40000000 ; 1 t: 128.11$

cs; for; cnt: $40000000 ; 1 t: 21$

cs; while; cnt: $40000000 ; 1 t: 19$

Cs; ForEach + lambda; cnt:40000000;lt:95

CS;ForEach + named function; cnt:40000000;1t:98

cs; Recursive; cnt: $40000000 ; 1 t: 102$

Java; for; cnt: $40000000 ; 1 t: 62$

Java; while; cnt: $40000000 ;$ lt: 49

Java; forEach; cnt: 40000000 ; lt:219

Java;ForEach + lambda; cnt:40000000;lt:138

Java; ForEach + named function; cnt:40000000; lt:2032

Java; Recursive; cnt:40000000;lt:89

Js; for; cnt: 40000000 ;lt: 36

Js; While; cnt: 40000000 ; lt: 34

Js; ForEach + lambda function; cnt: 40000000 ;lt: 246

Js; ForEach + named function; cnt: 40000000 ; lt: 239

Js; Recursive; cnt: 40000000 ; lt: 255

Pas;for; cnt: 40000000 ; lt: 83

Pas; while; cnt: 40000000 ; lt: 52

Pas; forEach; cnt:40000000; lt: 574

Pas;ForEach + lambda; cnt:40000000;lt: Not supported

Pas;ForEach + named function; cnt:40000000;lt: Not supported

Pas; Recursive; cnt:40000000;lt: 148

Py;For; cnt: 40000000 ; lt: 1175

Py; While; cnt: 40000000 ; lt: 1874

Py; ForEach [+ lambda]; cnt: 40000000 ; lt: 233

Py; ForEach + named function; cnt: 40000000 ; lt: 3260

Py; recursive; cnt: 40000000 ; lt: 9195

Cpp; while; cnt: $40000000 ;$ lt: 13.119

Cpp; do-while; cnt:40000000; lt:17.027

Cpp; for; cnt: $40000000 ; 1 t: 9.737$

Cpp; ForEach + lambda; cnt:40000000; lt:1119.74

Cpp;ForEach + named function; cnt:40000000;lt:1099.06

Cpp; Recursive; cnt:40000000; lt:128.921

cs; for; cnt: $40000000 ; 1 t: 21$

cs; while; cnt: $40000000 ; 1 t: 21$

CS;ForEach + lambda; cnt:40000000;1t:98

CS;ForEach + named function; cnt:40000000;1t:94

Cs; Recursive; cnt:40000000; lt:83

Java;for; cnt: $40000000 ; 1 t: 54$

Java; while; cnt: $40000000 ; 1 t: 42$

Java; forEach; cnt: 40000000 ; lt: 220

Java; ForEach + lambda; cnt:40000000; lt:174

Java;ForEach + named function; cnt:40000000;1t:1971

Java; Recursive; cnt:40000000; lt: 100

Js;for;cnt: 40000000 ; lt: 36

Js; While; cnt: 40000000 ; lt: 33

Js; ForEach + lambda function; cnt: 40000000 ;lt: 242

Js;ForEach + named function; cnt: 40000000 ; lt: 233

Js; Recursive; cnt: 40000000 ; lt: 284

Pas;for; cnt: 40000000 ; lt: 82

Pas; while; cnt:40000000; lt: 64

Pas; forEach; cnt:40000000; lt: 549

Pas; ForEach + lambda; cnt:40000000;lt: Not supported

Pas;ForEach + named function; cnt:40000000;lt: Not supported

Pas; Recursive; cnt:40000000; lt: 134

Py; For; cnt: 40000000 ; lt: 1145

Py; While; cnt: 40000000 ; it: 1776

Py; ForEach [+ lambda]; cnt: 40000000 ; lt: 729

Central-European Journal of New Technologies in Research, Education and Practice

Volume 2, Number 1, 2020. 
Py; ForEach + named function; cnt: 40000000 ; lt: 3322

Py; recursive; cnt: 40000000 ; lt: 8969

Cpp; while; cnt: $40000000 ;$ lt: 3.234

Cpp; do-while; cnt: $40000000 ; 1 t: 0.561$

Cpp; for; cnt:40000000; lt:4.021

Cpp; ForEach + lambda; cnt:40000000;1t:1119.4

Cpp; ForEach + named function; cnt:40000000;lt:1111.89

Cpp; Recursive; cnt: $40000000 ;$ lt:128.048

cs; for; cnt: $40000000 ; 1 t: 22$

cs; while; cnt: $40000000 ; 1 t: 19$

CS;ForEach + lambda; cnt:40000000;lt:94

CS;ForEach + named function; cnt:40000000;lt:91

cs; Recursive; cnt: $40000000 ;$ lt: 87

Java;for; cnt:40000000; lt:53

Java; while; cnt: $40000000 ;$ lt: 49

Java; forEach; cnt: $40000000 ; 1 t: 232$

Java;ForEach + lambda; cnt:40000000;lt:184

Java; ForEach + named function; cnt:40000000; lt:1972

Java; Recursive; cnt:40000000; lt: 100

Js; for; cnt: 40000000 ; lt: 36

Js; While; cnt: 40000000 ; lt: 33

Js; ForEach + lambda function; cnt: 40000000 ;lt: 243

Js;ForEach + named function; cnt: 40000000 ; lt: 230

Js; Recursive; cnt: 40000000 ; lt: 260

Pas;for;cnt:40000000;lt: 79

Pas; while; cnt:40000000;lt: 65

Pas; forEach; cnt:40000000; lt: 553

Pas;ForEach + lambda; cnt:40000000;lt: Not supported

Pas;ForEach + named function; cnt:40000000; lt: Not supported

Pas; Recursive; cnt:40000000; lt: 130

Py; For; cnt: 40000000 ;lt: 1162

Py; While; cnt: 40000000 ; lt: 2469

Py; ForEach [+ lambda]; cnt: 40000000 ; lt: 248

Py; ForEach + named function; cnt: 40000000 ; lt: 5494

Py; recursive; cnt: 40000000 ; lt: 9184

Cpp; while; cnt: $40000000 ; 1 t: 0.22$

Cpp; do-while; cnt:40000000; lt:0.463

Cpp; for; cnt: $40000000 ; 1 t: 5.005$

Cpp;ForEach + lambda; cnt:40000000;lt:1107.88

Cpp;ForEach + named function; cnt:40000000;1t:1121.43

Cpp; Recursive; cnt: 40000000 ; lt:131.073

CS; for; cnt: $40000000 ;$ it: 21

cs; while; cnt: $40000000 ; 1 t: 22$

Cs; ForEach + lambda; cnt:40000000;1t:93

CS; ForEach + named function; cnt:40000000;lt:92

cs; Recursive; cnt: $40000000 ; 1 t: 93$

Java; for; cnt: $40000000 ; 1 t: 36$

Java; while; cnt: $40000000 ; 1 t: 53$

Java; forEach; cnt: $40000000 ; 1 t: 225$

Java; ForEach + lambda; cnt:40000000;lt:159

Java; ForEach + named function; cnt:40000000;lt:1950

Java; Recursive; cnt:40000000;lt:95

Js; for; cnt: 40000000 ; lt: 32

Js; While; cnt: 40000000 ; lt: 31

Js; ForEach + lambda function; cnt: 40000000 ; lt: 250

Js;ForEach + named function; cnt: 40000000 ; lt: 236

Js; Recursive; cnt: 40000000 ; lt: 263

Pas; for; cnt:40000000;lt: 82

Pas; while; cnt:40000000;lt: 67

Pas;forEach; cnt: $40000000 ;$ it: 546

Pas;ForEach + lambda; cnt:40000000;lt:Not supported

Pas;ForEach + named function; cnt:40000000; lt: Not supported

Central-European Journal of New Technologies in Research, Education and Practice

Volume 2, Number 1, 2020. 
Pas; Recursive; cnt: 40000000 ; lt:

Py;For; cnt: 40000000 ; lt: 1197

Py; While; cnt: 40000000 ; it: 1660

Py; ForEach [+ lambda]; cnt: 40000000 ; lt: 47

Py; ForEach + named function; cnt: 40000000 ; lt: 2791

Py; recursive; cnt: 40000000 ; lt: 8820

Cpp; while; cnt: $40000000 ; 1 t: 0.233$

Cpp; do-while; cnt: $40000000 ; 1 t: 2.049$

Cpp; for; cnt: 40000000 ; lt: 6.047

Cpp; ForEach + lambda; cnt:40000000;1t:1114.01

Cpp;ForEach + named function; cnt:40000000;lt:1118.2

Cpp; Recursive; cnt:40000000; lt:133.1

cs; for; cnt: $40000000 ;$ lt: 29

cs; while; cnt: $40000000 ; 1 t: 23$

Cs;ForEach + lambda; cnt:40000000;lt:92

cs; ForEach + named function; cnt:40000000;1t:92

cs; Recursive; cnt:40000000; it:86

Java;for; cnt: $40000000 ; 1 t: 55$

Java; while; cnt: 40000000 ; lt: 52

Java; forEach; cnt: $40000000 ; 1 t: 224$

Java;ForEach + lambda; cnt:40000000;lt:161

Java; ForEach + named function; cnt:40000000;lt:1966

Java; Recursive; cnt:40000000; lt:117

Js; for; cnt: 40000000 ; lt: 36

Js; While; cnt: 40000000 ; It: 35

Js;ForEach + lambda function; cnt: 40000000 ; lt: 244

Js; ForEach + named function; cnt: 40000000 ; lt: 235

Js; Recursive; cnt: 40000000 ; lt: 261

Pas;for;cnt:40000000; lt: 79

Pas; while; cnt:40000000;lt: 64

Pas; forEach; cnt:40000000; lt: 550

Pas;ForEach + lambda; cnt:40000000;lt: Not supported

Pas;ForEach + named function; cnt:40000000;lt: Not supported

Pas; Recursive; cnt:40000000; it: 137

Py; For; cnt: 40000000 ; lt: 81

Py; While; cnt: 40000000 ; lt: 2897

Py; ForEach [+ lambda]; cnt: 40000000 ; lt: 216

Py; ForEach + named function; cnt: 40000000 ; lt: 3273

Py; recursive; cnt: 40000000 ; lt: 11002

Cpp; while; cnt:40000000; lt: 1.006

Cpp; do-while; cnt:40000000; lt:0.084

cpp; for; cnt:40000000; lt:2.409

Cpp;ForEach + lambda; cnt:40000000;lt:1114.54

Cpp;ForEach + named function; cnt:40000000;lt:1099.17

Cpp; Recursive; cnt: $40000000 ;$ lt: 128.804

cs; for; cnt: $40000000 ; 1 t: 22$

cs; while; cnt: $40000000 ; 1 t: 19$

CS; ForEach + lambda; cnt:40000000; lt:94

CS; ForEach + named function; cnt:40000000;1t:89

cs; Recursive; cnt: $40000000 ; 1 t: 86$

Java; for; cnt:40000000; lt:54

Java; while; cnt:40000000; it:51

Java; forEach; cnt:40000000; lt:209

Java; ForEach + lambda; cnt:40000000; lt:160

Java; ForEach + named function; cnt:40000000; lt:1966

Java; Recursive; cnt:40000000; lt:113

Js;for;cnt: 40000000 ; lt: 36

Js; While; cnt: 40000000 ; It: 35

Js; ForEach + lambda function; cnt: 40000000 ; lt: 241

Js;ForEach + named function; cnt: 40000000 ; lt: 234

Js; Recursive; cnt: 40000000 ; lt: 257

Pas;for; cnt:40000000; it: 79

Central-European Journal of New Technologies in Research, Education and Practice

Volume 2, Number 1, 2020. 
Pas; while; cnt:40000000; lt: 67

Pas; forEach; cnt:40000000; lt: 545

Pas;ForEach + lambda; cnt:40000000;lt: Not supported

Pas;ForEach + named function; cnt:40000000;lt: Not supported

Pas; Recursive; cnt: 40000000 ; lt: 134

Py; For; cnt: 40000000 ; lt: 461

Py; While; cnt: 40000000 ; lt: 2933

Py; ForEach [+ lambda]; cnt: 40000000 ; lt: 1159

Py; ForEach + named function; cnt: 40000000 ; lt: 3164

Py; recursive; cnt: 40000000 ; lt: 11564

Cpp; while; cnt: 40000000 ; lt:0.98

Cpp; do-while; cnt: $40000000 ; 1 t: 2.854$

Cpp; for; cnt: $40000000 ; 1 t: 6.992$

Cpp; ForEach + lambda; cnt:40000000;lt:1116.53

Cpp; ForEach + named function; cnt:40000000;1t:1115.34

Cpp; Recursive; cnt: 40000000 ; lt:127.015

Cs; for; cnt: $40000000 ; 1 t: 38$

cs; while; cnt: $40000000 ;$ lt: 8

CS;ForEach + lambda; cnt:40000000;lt:102

cs;ForEach + named function; cnt:40000000;lt:93

cs; Recursive; cnt:40000000; lt:80

Java; for; cnt: $40000000 ;$ lt: 64

Java; while; cnt:40000000; it: 49

Java; forEach; cnt: 40000000 ; lt: 210

Java;ForEach + lambda; cnt:40000000;1t:157

Java; ForEach + named function; cnt:40000000;lt:1985

Java; Recursive; cnt:40000000; lt:105

Js; for; cnt: 40000000 ; lt: 36

Js; While; cnt: 40000000 ; It: 35

Js; ForEach + lambda function; cnt: 40000000 ;lt: 240

Js; ForEach + named function; cnt: 40000000 ; lt: 233

Js; Recursive; cnt: 40000000 ; lt: 264

Pas; for; cnt: 40000000 ; lt: $\quad 80$

Pas; while; cnt: 40000000 ; lt: 68

Pas; forEach; cnt:40000000; it: 557

Pas;ForEach + lambda; cnt:40000000; lt: Not supported

Pas;ForEach + named function; cnt:40000000;lt:Not supported

Pas; Recursive; cnt:40000000; lt: 147

Py;For; cnt: 40000000 ; lt: 1180

Py; While; cnt: 40000000 ; it: 1764

Py; ForEach [+ lambda]; cnt: 40000000 ; lt: 320

Py; ForEach + named function; cnt: 40000000 ; lt: 3195

Py; recursive; cnt: 40000000 ; lt: 9001

Cpp; while; cnt:40000000; lt:2.975

Cpp; do-while; cnt: $40000000 ; 1 t: 3.126$

Cpp; for; cnt: 40000000 ; lt: 4.612

Cpp; ForEach + lambda; cnt:40000000;1t:1122.08

Cpp; ForEach + named function; cnt:40000000;lt:1115.68

Cpp; Recursive; cnt: $40000000 ;$ lt:125.834

Cs; for; cnt: $40000000 ; 1 t: 24$

cs; while; cnt: $40000000 ; 1 t: 19$

CS; ForEach + lambda; cnt:40000000;1t:91

cs; ForEach + named function; cnt:40000000;1t:89

cs; Recursive; cnt:40000000; it:86

Java; for; cnt: $40000000 ; 1 t: 37$

Java; while; cnt: $40000000 ; 1 t: 47$

Java; forEach; cnt: $40000000 ; 1 t: 222$

Java;ForEach + lambda; cnt:40000000;lt:148

Java;ForEach + named function; cnt:40000000;lt:1964

Java; Recursive; cnt:40000000; lt:102

Js; for; cnt: 40000000 ; lt: 36

Js; While; cnt: 40000000 ; It: 33

Central-European Journal of New Technologies in Research, Education and Practice

Volume 2, Number 1, 2020. 
Js; ForEach + lambda function; cnt: 40000000 ;lt: 240

Js; ForEach + named function; cnt: 40000000 ; lt: 232

Js; Recursive; cnt: 40000000 ; lt: 262

Pas;for;cnt:40000000; it: 82

Pas; while; cnt:40000000;lt: 64

Pas; forEach; cnt:40000000;lt: 552

Pas;ForEach + lambda; cnt:40000000;lt: Not supported

Pas;ForEach + named function; cnt:40000000; lt: Not supported

Pas; Recursive; cnt:40000000; lt: 135

Py;For; cnt: 40000000 ; lt: 1146

Py; While; cnt: 40000000 ; lt: 1817

Py; ForEach [+ lambda]; cnt: 40000000 ; lt: 193

Py; ForEach + named function; cnt: 40000000 ; lt: 3313

Py; recursive; cnt: 40000000 ; lt: 11275

Cpp; while; cnt: $40000000 ; 1 t: 3.112$

Cpp; do-while; cnt:40000000; lt:1.315

Cpp; for; cnt: $40000000 ; 1 t: 3.389$

Cpp; ForEach + lambda; cnt:40000000;lt:1113.93

Cpp; ForEach + named function; cnt:40000000;lt:1119.23

Cpp; Recursive; cnt:40000000; lt:128.719

cs; for; cnt: $40000000 ; 1 t: 21$

cs; while; cnt: $40000000 ; 1 t: 19$

CS; ForEach + lambda; cnt:40000000; lt:92

CS; ForEach + named function; cnt:40000000;1t:92

cs; Recursive; cnt:40000000; 1t:87

Java;for; cnt: $40000000 ; 1 t: 55$

Java; while; cnt: $40000000 ;$ it: 50

Java; forEach; cnt:40000000; lt:241

Java; ForEach + lambda; cnt:40000000;lt:159

Java; ForEach + named function; cnt:40000000; lt:1982

Java; Recursive; cnt:40000000; lt:104

Js; for; cnt: 40000000 ; lt: 37

Js; While; cnt: 40000000 ; lt: 30

Js; ForEach + lambda function; cnt: 40000000 ; lt: 244

Js;ForEach + named function; cnt: 40000000 ;lt: 231

Js; Recursive; cnt: 40000000 ; lt: 261

Pas;for;cnt:40000000; lt: 87

Pas; while; cnt:40000000;it: 66

Pas; forEach; cnt:40000000;it: 554

Pas; ForEach + lambda; cnt:40000000; lt: Not supported

Pas;ForEach + named function; cnt:40000000;lt: Not supported

Pas; Recursive; cnt:40000000; lt: 133

Py; For; cnt: 40000000 ; lt: 1135

Py; While; cnt: 40000000 ; it: 1383

Py; ForEach [+ lambda]; cnt: 40000000 ; lt: 194

Py; ForEach + named function; cnt: 40000000 ; lt: 2675

Py; recursive; cnt: 40000000 ; lt: 8004

Cpp; while; cnt:40000000; lt: 7.681

Cpp; do-while; cnt: $40000000 ; 1 t: 1.819$

Cpp; for; cnt: $40000000 ; 1 t: 6.874$

Cpp;ForEach + lambda; cnt:40000000;1t:1107.76

Cpp; ForEach + named function; cnt:40000000;1t:1107.76

Cpp; Recursive; cnt:40000000; lt:140.189

cs; for; cnt: $40000000 ; 1 t: 14$

cs; while; cnt: $40000000 ; 1 t: 24$

CS; ForEach + lambda; cnt:40000000;lt:245

cs; ForEach + named function; cnt:40000000; lt:549

cs; Recursive; cnt:40000000; it:86

Java;for; cnt: $40000000 ; 1 t: 37$

Java; while; cnt:40000000; lt:59

Java; forEach; cnt: $40000000 ; 1 t: 224$

Java;ForEach + lambda; cnt:40000000; lt:153

Central-European Journal of New Technologies in Research, Education and Practice

Volume 2, Number 1, 2020. 
Java;ForEach + named function; cnt:40000000; lt:1981

Java; Recursive; cnt:40000000; lt:109

Js; for; cnt: 40000000 ; lt: 36

Js; While; cnt: 40000000 ; lt: 33

Js; ForEach + lambda function; cnt: 40000000 ; lt: 241

Js;ForEach + named function; cnt: 40000000 ; lt: 233

Js; Recursive; cnt: 40000000 ; lt: 259

Pas;for; cnt: 40000000 ; lt: 82

Pas; while; cnt: 40000000 ; lt: 64

Pas;forEach; cnt: 40000000 ; lt: 549

Pas;ForEach + lambda; cnt:40000000; lt: Not supported

Pas;ForEach + named function; cnt:40000000;lt: Not supported

Pas; Recursive; cnt:40000000;lt: 133

Py; For; cnt: 40000000 ; lt: 1131

Py; While; cnt: 40000000 ; lt: 1825

Py; ForEach [+ lambda]; cnt: 40000000 ; lt: 664

Py; ForEach + named function; cnt: 40000000 ; lt: 3704

Py; recursive; cnt: 40000000 ; lt: 10454

Cpp; while; cnt: $40000000 ; 1 t: 1.2$

Cpp;do-while; cnt:40000000;lt:1.137

Cpp; for; cnt: $40000000 ;$ it:0.599

Cpp; ForEach + lambda; cnt:40000000;lt:1111.89

Cpp;ForEach + named function; cnt:40000000; lt:1113.95

Cpp; Recursive; cnt:40000000; it:127.868

Cs; for; cnt: $40000000 ; 1 t: 29$

cs; while; cnt: 40000000 ; lt: 19

cs; ForEach + lambda; cnt:40000000; lt:94

CS;ForEach + named function; cnt:40000000;1t:91

cs; Recursive; cnt:40000000; lt: 93

Java; for; cnt: $40000000 ; 1 t: 23$

Java; while; cnt: $40000000 ; 1 t: 36$

Java; forEach; cnt:40000000; lt:203

Java;ForEach + lambda; cnt:40000000;lt:158

Java;ForEach + named function; cnt:40000000; lt:1969

Java; Recursive; cnt:40000000; lt:114

Js;for; cnt: 40000000 ;lt: 36

Js; While; cnt: 40000000 ; lt: 33

Js;ForEach + lambda function; cnt: 40000000 ;lt: 243

Js; ForEach + named function; cnt: 40000000 ; lt: 230

Js; Recursive; cnt: 40000000 ; lt: 261

Pas; for; cnt: 40000000 ; lt: 90

Pas; while; cnt: 40000000 ; lt: $\quad 60$

Pas; forEach; cnt:40000000; lt: 576

Pas; ForEach + lambda; cnt:40000000;lt:Not supported

Pas;ForEach + named function; cnt:40000000;lt:Not supported

Pas;Recursive; cnt:40000000;lt: 126

Py; For; cnt: 40000000 ; lt: 1141

Py; While; cnt: 40000000 ; lt: 1847

Py; ForEach [+ lambda]; cnt: 40000000 ; lt: 52

Py; ForEach + named function; cnt: 40000000 ; lt: 3655

Py; recursive; cnt: 40000000 ; lt: 8668

Cpp; while; cnt: $40000000 ; 1 t: 4.09$

Cpp; do-while; cnt: $40000000 ; 1 t: 2.544$

Cpp; for; cnt: $40000000 ; 1 t: 6.484$

Cpp; ForEach + lambda; cnt:40000000;1t:1115.32

Cpp; ForEach + named function; cnt:40000000;lt:1108.97

Cpp; Recursive; cnt:40000000; lt:110.164

Cs; for; cnt: $40000000 ; 1 t: 16$

cs; while; cnt: $40000000 ; 1 t: 31$

CS; ForEach + lambda; cnt:40000000;lt:113

CS;ForEach + named function; cnt:40000000;1t:81

cs; Recursive; cnt: 40000000 ; 1t: 89

Central-European Journal of New Technologies in Research, Education and Practice

Volume 2, Number 1, 2020. 
Java; for; cnt:40000000; lt:51

Java; while; cnt: $40000000 ; 1 t: 63$

Java; forEach; cnt: $40000000 ; 1 t: 220$

Java; ForEach + lambda; cnt:40000000; lt:148

Java;ForEach + named function; cnt:40000000; lt:1965

Java; Recursive; cnt: $40000000 ; 1 t: 115$

Js; for; cnt: 40000000 ;lt: 36

Js; While; cnt: 40000000 ; lt: 35

Js;ForEach + lambda function; cnt: 40000000 ;lt: 243

Js;ForEach + named function; cnt: 40000000 ;lt: 256

Js; Recursive; cnt: 40000000 ; lt: 253

Pas;for;cnt:40000000;lt: 90

Pas; while; cnt: 40000000 ; lt: 66

Pas; forEach; cnt: 40000000 ; lt: 538

Pas;ForEach + lambda; cnt:40000000;lt:Not supported

Pas;ForEach + named function; cnt:40000000;lt: Not supported

Pas;Recursive; cnt:40000000;lt: 135

Py; For; cnt: 40000000 ; lt: 1190

Py; While; cnt: 40000000 ; lt: 1833

Py; ForEach [+ lambda]; cnt: 40000000 ; lt: 270

Py; ForEach + named function; cnt: 40000000 ; lt: 4329

Py; recursive; cnt: 40000000 ; lt: 9364

Cpp; while; cnt: $40000000 ; 1 t: 16.581$

Cpp;do-while; cnt:40000000; lt:10.326

Cpp; for; cnt:40000000; it:17.516

Cpp;ForEach + lambda; cnt:40000000;1t:1101.75

Cpp; ForEach + named function; cnt:40000000;1t:1132.63

Cpp; Recursive; cnt: $40000000 ;$ lt:135.849

Cs; for; cnt: $40000000 ; 1 t: 26$

cs; while; cnt: $40000000 ; 1 t: 31$

cs; ForEach + lambda; cnt:40000000;1t:101

CS;ForEach + named function; cnt:40000000;1t:90

Cs; Recursive; cnt: $40000000 ; 1 t: 88$

Java; for; cnt: $40000000 ; 1 t: 40$

Java; while; cnt:40000000; lt:60

Java; forEach; cnt:40000000; lt:196

Java; ForEach + lambda; cnt:40000000;lt:201

Java; ForEach + named function; cnt:40000000; lt:1931

Java; Recursive; cnt: 40000000 ; lt: 108

Js; for; cnt: 40000000 ; lt: 35

Js; While; cnt: 40000000 ; lt: 32

Js; ForEach + lambda function; cnt: 40000000 ; lt: 251

Js; ForEach + named function; cnt: 40000000 ; it: 239

Js; Recursive; cnt: 40000000 ; lt: 260

Pas;for;cnt:40000000;lt: 76

Pas; while; cnt:40000000; lt: 65

Pas;forEach; cnt:40000000; it: 561

Pas;ForEach + lambda; cnt:40000000;1t:Not supported

Pas;ForEach + named function; cnt:40000000; lt: Not supported

Pas;Recursive; cnt:40000000; it: 126

Py; For; cnt: 40000000 ; lt: 2125

Py; While; cnt: 40000000 ;lt: 1330

Py; ForEach [+ lambda]; cnt: 40000000 ; lt: 2215

Py; ForEach + named function; cnt: 40000000 ; lt: 4767

Py; recursive; cnt: 40000000 ; lt: 9615

Cpp; while; cnt: $40000000 ; 1 t: 9.26$

Cpp; do-while; cnt:40000000; lt: 7.192

Cpp; for; cnt: $40000000 ; 1 t: 2.262$

Cpp;ForEach + lambda; cnt:40000000;1t:1122.75

Cpp; ForEach + named function; cnt:40000000; lt:1137.29

Cpp; Recursive; cnt:40000000; lt:123.278

Cs; for; cnt: $40000000 ; 1 t: 18$

Central-European Journal of New Technologies in Research, Education and Practice

Volume 2, Number 1, 2020. 


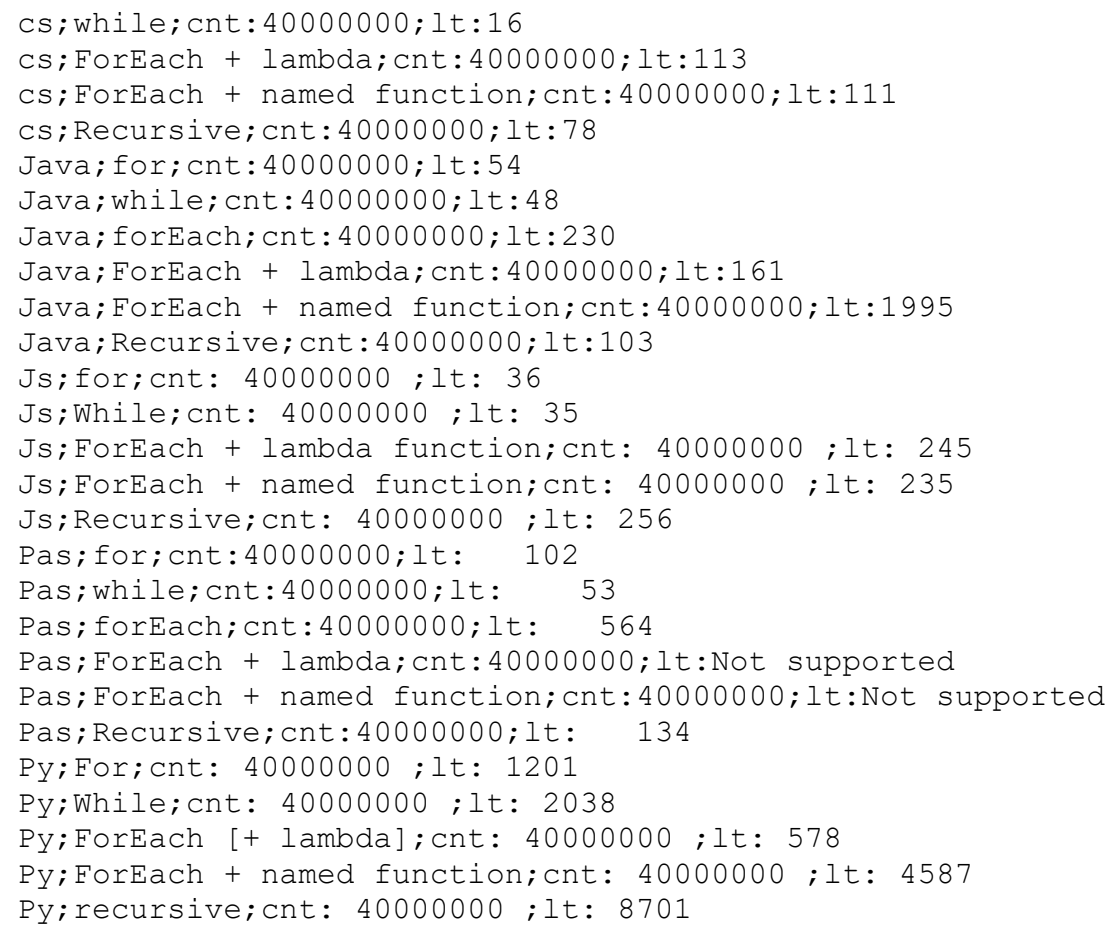

Central-European Journal of New Technologies in Research, Education and Practice 
Cpp;ForEach + lambda; cnt:40000000;1t:1127.67

Cpp;ForEach + named function; cnt:40000000;1t:1130.66

Cpp; Recursive; cnt: 40000000 ; lt:118.777

Cs; for; cnt: $40000000 ; 1$ t: 17

cs; while; cnt: $40000000 ;$ it: 14

CS; ForEach + lambda; cnt:40000000;lt:93

CS; ForEach + named function; cnt:40000000;lt:89

cs; Recursive; cnt:40000000; lt:95

Java; for; cnt: $40000000 ; 1 t: 45$

Java; while; cnt:40000000; lt: 58

Java; forEach; cnt:40000000; lt:216

Java; ForEach + lambda; cnt:40000000; lt:175

Java; ForEach + named function; cnt:40000000;lt:1961

Java; Recursive; cnt:40000000; lt:99

Js; for; cnt: 40000000 ; lt: 37

Js; While; cnt: 40000000 ; lt: 32

Js; ForEach + lambda function; cnt: 40000000 ; lt: 248

Js; ForEach + named function; cnt: 40000000 ;lt: 259

Js; Recursive; cnt: 40000000 ; lt: 258

Pas;for;cnt:40000000;lt: 79

Pas; while; cnt:40000000;lt: 68

Pas; forEach; cnt:40000000; lt: 528

Pas;ForEach + lambda; cnt:40000000;lt: Not supported

Pas;ForEach + named function; cnt:40000000;lt: Not supported

Pas; Recursive; cnt:40000000;lt: 135

Py;For; cnt: 40000000 ; lt: 3913

Py; While; cnt: 40000000 ;lt: 1955

Py; ForEach [+ lambda]; cnt: 40000000 ; lt: 262

Py; ForEach + named function; cnt: 40000000 ; lt: 3428

Py; recursive; cnt: 40000000 ;it: 9675

Cpp; while; cnt:40000000; lt: 6.028

Cpp;do-while; cnt:40000000; it:1.992

Cpp; for; cnt: $40000000 ; 1 t: 3.412$

Cpp;ForEach + lambda; cnt:40000000;1t:1157.15

Cpp; ForEach + named function; cnt:40000000;lt:1125.75

Cpp; Recursive; cnt:40000000; lt:130.32

cs; for; cnt: $40000000 ; 1 t: 21$

cs; while; cnt: $40000000 ; 1 t: 16$

cs; ForEach + lambda; cnt:40000000;lt:99

CS; ForEach + named function; cnt:40000000;lt:92

cs; Recursive; cnt: $40000000 ; 1 t: 90$

Java; for; cnt: $40000000 ;$ lt:53

Java; while; cnt:40000000; lt:50

Java; forEach; cnt: $40000000 ; 1 t: 218$

Java; ForEach + lambda; cnt:40000000; lt:164

Java; ForEach + named function; cnt:40000000;lt:1934

Java; Recursive; cnt:40000000; lt:111

Js; for; cnt: 40000000 ; lt: 36

Js; While; cnt: 40000000 ; lt: 35

Js;ForEach + lambda function; cnt: 40000000 ; lt: 258

Js;ForEach + named function; cnt: 40000000 ; lt: 243

Js; Recursive; cnt: 40000000 ; lt: 256

Pas; for; cnt: 40000000 ; lt: 78

Pas; while; cnt:40000000; lt: 65

Pas; forEach; cnt: 40000000 ; lt: 559

Pas;ForEach + lambda; cnt:40000000;lt: Not supported

Pas;ForEach + named function; cnt:40000000;lt: Not supported

Pas; Recursive; cnt:40000000;lt: 137

Py;For; cnt: 40000000 ; lt: 1207

Py; While; cnt: 40000000 ; it: 1839

Py; ForEach [+ lambda]; cnt: 40000000 ; lt: 258

Py; ForEach + named function; cnt: 40000000 ; lt: 3196

Py; recursive; cnt: 40000000 ; lt: 9268

Central-European Journal of New Technologies in Research, Education and Practice

Volume 2, Number 1, 2020. 
Cpp; while; cnt: $40000000 ; 1 t: 0.358$

Cpp; do-while; cnt: $40000000 ; 1 t: 2.786$

Cpp; for; cnt: $40000000 ; 1 t: 3.322$

Cpp; ForEach + lambda; cnt:40000000;lt:1107.68

Cpp;ForEach + named function; cnt:40000000;lt:1109.93

Cpp; Recursive; cnt: 40000000 ; lt:149.029

cs; for; cnt: $40000000 ; 1 t: 23$

cs; while; cnt: $40000000 ; 1 t: 19$

Cs;ForEach + lambda; cnt:40000000;lt:96

CS;ForEach + named function; cnt:40000000;lt:92

cs; Recursive; cnt: $40000000 ; 1$ t: 87

Java; for; cnt: $40000000 ; 1 t: 37$

Java; while; cnt:40000000; it:51

Java; forEach; cnt: $40000000 ; 1 t: 218$

Java; ForEach + lambda; cnt:40000000; lt:160

Java;ForEach + named function; cnt:40000000;1t:1983

Java; Recursive; cnt:40000000; lt:96

Js; for; cnt: 40000000 ; lt: 36

Js; While; cnt: 40000000 ; lt: 33

Js; ForEach + lambda function; cnt: 40000000 ;lt: 243

Js; ForEach + named function; cnt: 40000000 ; lt: 239

Js; Recursive; cnt: 40000000 ; lt: 259

Pas;for;cnt:40000000;lt: 82

Pas; while; cnt: 40000000 ; lt: 68

Pas; forEach; cnt:40000000;lt: 556

Pas;ForEach + lambda; cnt:40000000;lt: Not supported

Pas;ForEach + named function; cnt:40000000; lt: Not supported

Pas;Recursive; cnt:40000000;lt: 133

Py;For; cnt: 40000000 ; lt: 1177

Py; While; cnt: 40000000 ; lt: 1822

Py; ForEach [+ lambda]; cnt: 40000000 ; lt: 180

Py; ForEach + named function; cnt: 40000000 ; lt: 3663

Py; recursive; cnt: 40000000 ; lt: 9222

Cpp; while; cnt: $40000000 ; 1 t: 5.169$

Cpp; do-while; cnt:40000000;lt:3.163

Cpp; for; cnt: $40000000 ; 1 t: 0.998$

Cpp;ForEach + lambda; cnt:40000000;1t:1135.57

Cpp; ForEach + named function; cnt:40000000;lt:1123.54

Cpp; Recursive; cnt: $40000000 ; 1 t: 129.678$

cs; for; cnt: $40000000 ; 1 t: 21$

cs; while; cnt: $40000000 ; 1 t: 21$

cs; ForEach + lambda; cnt:40000000;lt:79

CS; ForEach + named function; cnt:40000000;lt:137

cs; Recursive; cnt: $40000000 ; 1 t: 90$

Java; for; cnt: $40000000 ; 1 t: 54$

Java; while; cnt:40000000; lt:54

Java; forEach; cnt: $40000000 ; 1 t: 235$

Java;ForEach + lambda; cnt:40000000;lt:161

Java; ForEach + named function; cnt:40000000;lt:1965

Java; Recursive; cnt:40000000; lt: 157

Js;for; cnt: 40000000 ; lt: 36

Js; While; cnt: 40000000 ; lt: 33

Js; ForEach + lambda function; cnt: 40000000 ; lt: 243

Js;ForEach + named function; cnt: 40000000 ;lt: 237

Js; Recursive; cnt: $40000000 ;$ it: 255

Pas; for; cnt: 40000000 ; lt: 82

Pas; while; cnt:40000000;it: 60

Pas; forEach; cnt:40000000;lt: 536

Pas;ForEach + lambda; cnt:40000000;lt:Not supported

Pas;ForEach + named function; cnt:40000000; lt: Not supported

Pas;Recursive; cnt:40000000;lt: 135

Py; For; cnt: 40000000 ; lt: 100

Central-European Journal of New Technologies in Research, Education and Practice

Volume 2, Number 1, 2020. 
Py; While; cnt: 40000000 ; it: 1208

Py; ForEach [+ lambda]; cnt: 40000000 ; lt: 498

Py; ForEach + named function; cnt: 40000000 ; lt: 3102

Py; recursive; cnt: 40000000 ; lt: 9127

Cpp; while; cnt:40000000; lt:3.758

Cpp; do-while; cnt:40000000;lt:2.042

Cpp; for; cnt: $40000000 ; 1 t: 6.67$

Cpp; ForEach + lambda; cnt:40000000;lt:1093.56

Cpp; ForEach + named function; cnt:40000000;lt:889.726

Cpp; Recursive; cnt: $40000000 ;$ lt:236.729

cs; for; cnt: $40000000 ; 1 t: 12$

cs; while; cnt: $40000000 ; 1 t: 24$

CS;ForEach + lambda; cnt:40000000;1t:264

CS; ForEach + named function; cnt:40000000;lt:141

cs; Recursive; cnt: $40000000 ; 1 t: 73$

Java;for; cnt: $40000000 ; 1 t: 53$

Java; while; cnt:40000000; lt:51

Java; forEach; cnt: $40000000 ; 1 t: 313$

Java;ForEach + lambda; cnt:40000000;lt:197

Java;ForEach + named function; cnt:40000000;1t:2064

Java; Recursive; cnt:40000000; lt:106

Js;for; cnt: 40000000 ; lt: 38

Js; While; cnt: 40000000 ; lt: 32

Js;ForEach + lambda function; cnt: 40000000 ; lt: 246

Js;ForEach + named function; cnt: 40000000 ; lt: 239

Js; Recursive; cnt: 40000000 ; lt: 264

Pas;for; cnt: 40000000 ; lt: 90

Pas; while; cnt:40000000;lt: 67

Pas;forEach; cnt:40000000; lt: 558

Pas; ForEach + lambda; cnt:40000000;1t: Not supported

Pas;ForEach + named function; cnt:40000000;lt:Not supported

Pas;Recursive; cnt:40000000; lt: 135

Py;For; cnt: 40000000 ; lt: 1199

Py; While; cnt: 40000000 ; lt: 1826

Py; ForEach [+ lambda]; cnt: 40000000 ; lt: 285

Py; ForEach + named function; cnt: 40000000 ; lt: 3359

Py; recursive; cnt: 40000000 ; lt: 11310

Cpp; while; cnt:40000000; lt:4.049

Cpp; do-while; cnt:40000000;lt:1.115

Cpp; for; cnt: $40000000 ; 1 t: 20.573$

Cpp;ForEach + lambda; cnt:40000000;lt:1223.32

Cpp; ForEach + named function; cnt:40000000;lt:1120.09

Cpp; Recursive; cnt: $40000000 ; 1 t: 128.421$

cs; for; cnt: 40000000 ; lt: 9

cs; while; cnt: $40000000 ; 1 t: 18$

cs; ForEach + lambda; cnt:40000000;lt:130

CS;ForEach + named function; cnt:40000000;1t:99

cs; Recursive; cnt: $40000000 ; 1 t: 88$

Java; for; cnt: $40000000 ; 1 t: 54$

Java; while; cnt: $40000000 ; 1 t: 51$

Java; forEach; cnt: 40000000 ; lt:216

Java;ForEach + lambda; cnt:40000000; lt:190

Java; ForEach + named function; cnt:40000000; lt:1966

Java; Recursive; cnt:40000000;lt:106

Js; for; cnt: $40000000 ;$ lt: 36

Js; While; cnt: 40000000 ; lt: 32

Js; ForEach + lambda function; cnt: 40000000 ; lt: 239

Js;ForEach + named function; cnt: 40000000 ; lt: 236

Js; Recursive; cnt: 40000000 ; lt: 260

Pas;for;cnt:40000000; lt: 83

Pas; while; cnt:40000000;it: 69

Pas; forEach; cnt:40000000; it: 553

Central-European Journal of New Technologies in Research, Education and Practice

Volume 2, Number 1, 2020. 
Pas;ForEach + lambda; cnt:40000000;lt: Not supported

Pas;ForEach + named function; cnt:40000000;lt: Not supported

Pas; Recursive; cnt: 40000000 ; lt:

Py; For; cnt: 40000000 ; lt: 1048

Py; While; cnt: 40000000 ; lt: 791

Py; ForEach [+ lambda]; cnt: $40000000 ; 1 t: 248$

Py; ForEach + named function; cnt: 40000000 ; lt: 3314

Py; recursive; cnt: 40000000 ; lt: 10663

Cpp; while; cnt:40000000; lt: 7.188

Cpp; do-while; cnt: $40000000 ; 1 t: 0.537$

Cpp; for; cnt: $40000000 ; 1 t: 12.518$

Cpp; ForEach + lambda; cnt:40000000;1t:1152.84

Cpp;ForEach + named function; cnt:40000000;1t:1147.2

Cpp; Recursive; cnt:40000000; lt:127.19

cs; for; cnt: $40000000 ;$ it: 28

cs; while; cnt: $40000000 ; 1 t: 24$

CS; ForEach + lambda; cnt:40000000;lt:107

CS; ForEach + named function; cnt:40000000;1t:91

Cs; Recursive; cnt:40000000; it:86

Java; for; cnt: $40000000 ; 1 t: 57$

Java; while; cnt: $40000000 ; 1 t: 42$

Java; forEach; cnt: $40000000 ; 1 t: 218$

Java; ForEach + lambda; cnt:40000000; lt:185

Java;ForEach + named function; cnt:40000000; lt:1984

Java; Recursive; cnt: $40000000 ; 1 t: 111$

Js; for; cnt: 40000000 ; lt: 36

Js; While; cnt: 40000000 ; lt: 34

Js; ForEach + lambda function; cnt: 40000000 ;lt: 250

Js;ForEach + named function; cnt: 40000000 ; lt: 241

Js; Recursive; cnt: 40000000 ; lt: 281

Pas;for; cnt:40000000;lt: 78

Pas; while; cnt:40000000;lt: 60

Pas; forEach; cnt: 40000000 ; lt: 550

Pas;ForEach + lambda; cnt:40000000;lt: Not supported

Pas;ForEach + named function; cnt:40000000;lt: Not supported

Pas; Recursive; cnt:40000000;lt: 120

Py; For; cnt: 40000000 ; lt: 1062

Py; While; cnt: 40000000 ;lt: 2101

Py; ForEach [+ lambda]; cnt: 40000000 ; lt: 293

Py;ForEach + named function; cnt: 40000000 ; lt: 3142

Py; recursive; cnt: 40000000 ; lt: 9430

Cpp; while; cnt:40000000; lt:9.256

Cpp; do-while; cnt:40000000;lt: 1.018

Cpp; for; cnt: $40000000 ; 1 t: 1.817$

Cpp; ForEach + lambda; cnt:40000000;1t:1123.14

Cpp; ForEach + named function; cnt:40000000; lt:1113.97

Cpp; Recursive; cnt: $40000000 ; 1 t: 129.866$

Cs; for; cnt: $40000000 ; 1 t: 21$

cs; while; cnt: 40000000 ; lt: 22

CS; ForEach + lambda; cnt:40000000;lt:104

CS;ForEach + named function; cnt:40000000;lt:92

cs; Recursive; cnt:40000000; 1t:100

Java;for; cnt: $40000000 ; 1 t: 60$

Java; while; cnt: $40000000 ; 1 t: 53$

Java; forEach; cnt: $40000000 ; 1 t: 234$

Java; ForEach + lambda; cnt:40000000;lt:162

Java; ForEach + named function; cnt:40000000; lt:1955

Java; Recursive; cnt:40000000; lt:111

Js; for; cnt: 40000000 ; lt: 36

Js; While; cnt: 40000000 ; lt: 34

Js; ForEach + lambda function; cnt: $40000000 ; 1 t: 240$

Js; ForEach + named function; cnt: 40000000 ; lt: 234

Central-European Journal of New Technologies in Research, Education and Practice

Volume 2, Number 1, 2020. 


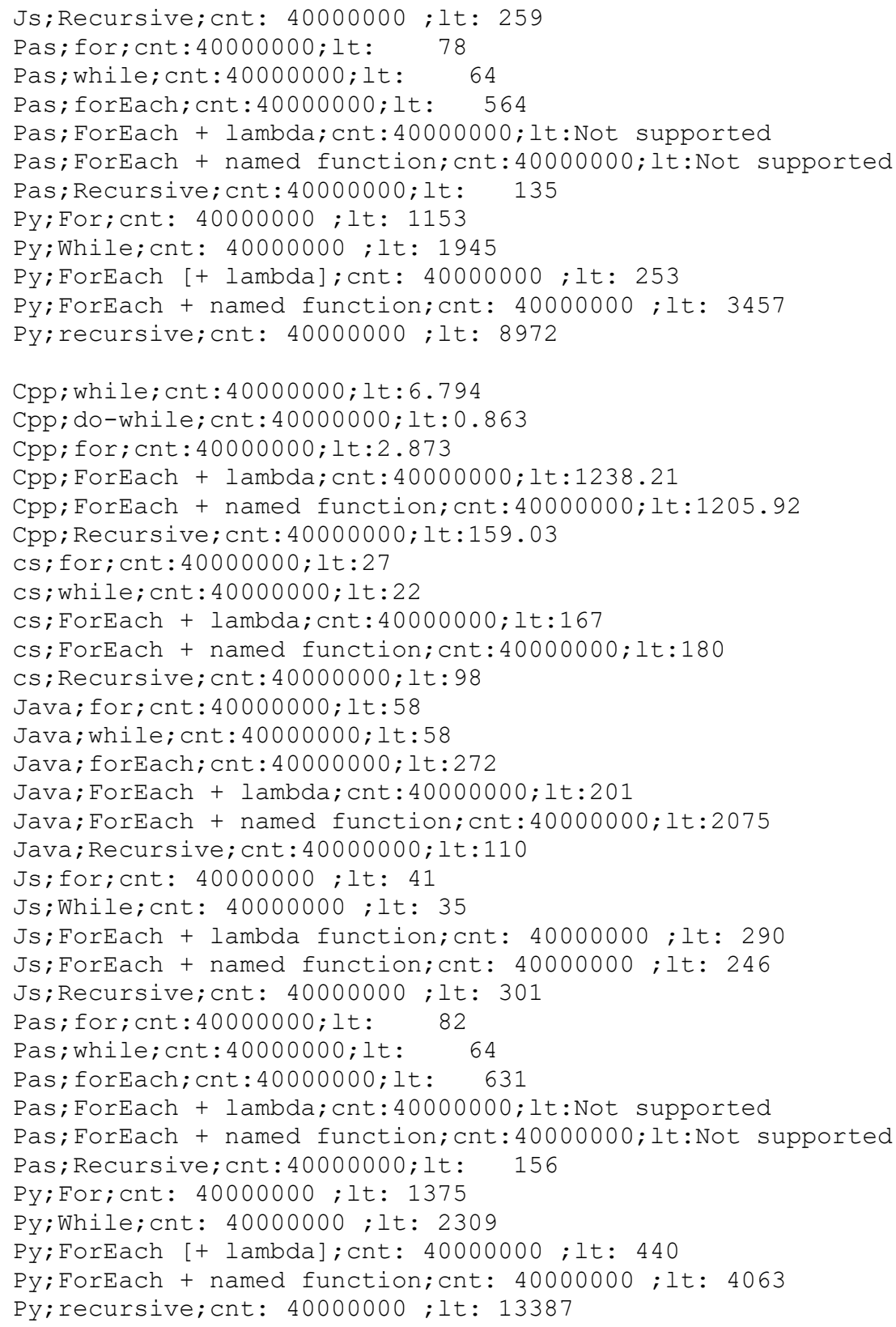

\section{Authors}

\section{MENYHÁRT László}

Eötvös Loránd University, Faculty of Informatics, Department of Media and Educational Informatics, Budapest, Hungary, e-mail: menyhart@inf.elte.hu

\section{About this document}

\author{
Published in:
}

CENTRAL-EUROPEAN JOURNAL OF NEW TECHNOLOGIES IN RESEARCH, EDUCATION AND PRACTICE

Volume 2, Number 1. 2020.

ISSN: 2676-9425 (online)

DOI:

10.36427/CEJNTREP.2.1.421

Central-European Journal of New Technologies in Research, Education and Practice 


\section{License}

Copyright (C) MENYHÁRT László, 2020.

Licensee CENTRAL-EUROPEAN JOURNAL OF NEW TECHNOLOGIES IN RESEARCH, EDUCATION AND PRACTICE, Hungary. This article is an open access article distributed under the terms and conditions of the Creative Commons Attribution (CC-BY) license.

http://creativecommons.org/licenses/by/4.0/

Central-European Journal of New Technologies in Research, Education and Practice

Volume 2, Number 1, 2020. 